\title{
A two-dimensional high-order well-balanced scheme for the shallow water equations with topography and Manning friction
}

\author{
Victor Michel-Dansac ${ }^{\mathrm{a}, *}$, Christophe Berthon ${ }^{\mathrm{b}}$, Stéphane Clain $^{\mathrm{c}, \mathrm{d}}$, Françoise Foucher ${ }^{\mathrm{b}, \mathrm{e}}$ \\ ${ }^{a}$ Université de Strasbourg, CNRS, Inria, IRMA, F-67000 Strasbourg, France \\ ${ }^{b}$ Laboratoire de Mathématiques Jean Leray, CNRS UMR 6629, Université de Nantes, 2 rue de la Houssinière, BP 92208 , 44322 \\ Nantes Cedex 3, France \\ ${ }^{c}$ CFUM - Centre of Physics, University of Minho, Azurém Campus, 4800-058 Guimarães, Portugal \\ ${ }^{d}$ DMAT - Department of Mathematics, University of Minho, Azurém Campus, 4800-058 Guimarães, Portugal \\ ${ }^{e}$ École Centrale de Nantes, 1 rue de La Noë, BP 9210144321 Nantes Cedex 3, France
}

\begin{abstract}
We develop a two-dimensional high-order numerical scheme that exactly preserves and captures the moving steady states of the shallow water equations with topography or Manning friction. The high-order accuracy relies on a suitable polynomial reconstruction, while the well-balancedness property is based on the first-order scheme from $[52,53]$, extended to two space dimensions. To get both properties, we use a convex combination between the highorder scheme and the first-order well-balanced scheme. By adequately choosing the convex combination parameter following a very simple steady state detector, we ensure that the resulting scheme is both high-order accurate and well-balanced. The method is then supplemented with a MOOD procedure to eliminate the spurious oscillations coming from the high-order polynomial reconstruction and to guarantee the physical admissibility of the solution. Numerical experiments show that the scheme indeed possesses the claimed properties. The simulation of the 2011 Tōhoku tsunami, on real data, further confirms the relevance of this technique.
\end{abstract}

Keywords: shallow water equations, Manning friction, high-order schemes, Godunov-type schemes, well-balanced schemes, moving steady states

2010 MSC: 65M08, 65M12

\section{Introduction}

This work is concerned with the numerical approximation of the shallow water equations, given in two space dimensions by:

$$
\left\{\begin{array}{l}
\partial_{t} h+\nabla \cdot \boldsymbol{q}=0 \\
\partial_{t} \boldsymbol{q}+\boldsymbol{\nabla} \cdot\left(\frac{\boldsymbol{q} \otimes \boldsymbol{q}}{h}+\frac{1}{2} g h^{2} \boldsymbol{I}\right)=-g h \boldsymbol{\nabla} Z-k \boldsymbol{q}\|\boldsymbol{q}\| h^{-\eta},
\end{array}\right.
$$

where $x$ and $y$ are the space variables, $t$ is the time variable, $h \geq 0$ is the water height, $\boldsymbol{q}=\left(q_{x}, q_{y}\right)^{\top}$ is the water discharge, with the ${ }^{\top}$ symbol indicating the transposition operation, and $g>0$ is the gravity constant. The

\footnotetext{
${ }^{*}$ Corresponding author

Email addresses: victor.michel-dansac@inria.fr (Victor Michel-Dansac), christophe.berthon@univ-nantes.fr (Christophe Berthon), clain@math.uminho.pt (Stéphane Clain), francoise.foucher@ec-nantes.fr (Françoise Foucher)
} 
homogeneous system is supplemented with two source terms. The topography source term is given by:

$$
\boldsymbol{S}^{t}=-g h \nabla Z
$$

where $Z(x)$ is the fixed topography function. In addition, the nonlinear Manning friction source term (see for instance [33]) is given by:

$$
\boldsymbol{S}^{f}=-k \boldsymbol{q}\|\boldsymbol{q}\| h^{-\eta}
$$

where $k \geq 0$ is the Manning friction coefficient and $\eta=7 / 3$. Other friction models exist in the hydraulic engineering literature, see for instance [27], Chapter 5. Examples include the Darcy-Weisbach or Chézy friction laws, presented in [33], which take the same form as (1.3), but with $\eta=2$ and a different value of $k$. Therefore, they also fall within the scope of this study. However, most hydraulic engineering models are only made to represent flows in one dimension, see for instance $[16,66,32,54]$. Even though these models have roughly the same shape as (1.3), we do not consider them here since they are less generic.

To shorten the notation, we rewrite (1.1) under the form

$$
\partial_{t} W+\partial_{x} F(W)+\partial_{y} G(W)=\left(\begin{array}{c}
0 \\
\boldsymbol{S}^{\boldsymbol{t}}(W)+\boldsymbol{S}^{\boldsymbol{f}}(W)
\end{array}\right),
$$

where the conserved variables $W$ and the physical fluxes $F(W)$ and $G(W)$ are given by:

$$
W=\left(\begin{array}{c}
h \\
q_{x} \\
q_{y}
\end{array}\right) ; \quad F(W)=\left(\begin{array}{c}
q_{x} \\
\frac{q_{x}^{2}}{h}+\frac{1}{2} g h^{2} \\
\frac{q_{x} q_{y}}{h}
\end{array}\right) ; \quad G(W)=\left(\begin{array}{c}
q_{y} \\
\frac{q_{x} q_{y}}{h} \\
\frac{q_{y}^{2}}{h}+\frac{1}{2} g h^{2}
\end{array}\right) .
$$

The admissible states space of the 2D shallow water equations is the following convex set:

$$
\Omega=\left\{W=(h, \boldsymbol{q})^{\top} \in \mathbb{R}^{3}, h \geq 0\right\},
$$

which accounts for dry areas when $h=0$. In addition, we define the water velocity $\boldsymbol{u}$ such that $\boldsymbol{q}=h \boldsymbol{u}$. In dry areas, i.e. when $h$ vanishes, we assume that both $\boldsymbol{u}$ and $\boldsymbol{S}^{\boldsymbol{f}}$ vanish.

In the context of numerical simulations, the preservation of the steady states of the shallow water equations, obtained by taking a vanishing time derivative in (1.1), is of prime importance. An example of these stationary solutions is the well-known lake at rest steady state, which is nothing but a steady solution with a vanishing discharge:

$$
\left\{\begin{array}{l}
\boldsymbol{q}=\mathbf{0}, \\
h+Z=\mathrm{cst} .
\end{array}\right.
$$

This steady state and its numerical preservation have been the object of much work in the last 25 years, we refer for instance to the non-exhaustive lists $[47,46,3,15,10]$ in one space dimension and $[4,37,67,31]$ in two space dimensions. The general 2D steady states with nonzero discharge, called moving steady states, are constrained with a vanishing discharge divergence, and their study is quite complex. In this manuscript, we only consider 
moving steady states in one space dimension.

With notation adapted from (1.1), the 1D shallow water equations read

$$
\left\{\begin{array}{l}
\partial_{t} h+\partial_{x} q=0 \\
\partial_{t} q+\partial_{x}\left(\frac{q^{2}}{h}+\frac{1}{2} g h^{2}\right)=-g h \partial_{x} Z-k q|q| h^{-\eta}
\end{array}\right.
$$

The 1D moving steady states, obtained by canceling the time derivatives in (1.8), are governed by:

$$
\left\{\begin{array}{l}
q=q_{0}, \\
\partial_{x}\left(\frac{q_{0}^{2}}{h}+\frac{1}{2} g h^{2}\right)=-g h \partial_{x} Z-k q|q| h^{-\eta},
\end{array}\right.
$$

where $q_{0}$, which can be nonzero, represents the discharge of the steady solution, which is constant in space and time.

Considering a vanishing friction, i.e. setting $k=0$ in (1.9), we recover the moving steady states with topography, governed by:

$$
\left\{\begin{array}{l}
q=q_{0}, \\
\partial_{x}\left(\frac{q_{0}^{2}}{h}+\frac{1}{2} g h^{2}\right)=-g h \partial_{x} Z .
\end{array}\right.
$$

If we additionally consider smooth data, the moving topography-only steady states turn out to be governed by the following algebraic relation, which is nothing but a statement of Bernoulli's principle:

$$
\frac{q_{0}^{2}}{2} \partial_{x}\left(\frac{1}{h^{2}}\right)+g \partial_{x}(h+Z)=0
$$

The numerical preservation of these steady states has also been the object of much work in the last two decades, see for instance $[42,20,8,52]$.

On a flat topography, but with a nonzero friction, we get the friction-only steady states, given by

$$
\left\{\begin{array}{l}
q=q_{0} \\
\partial_{x}\left(\frac{q_{0}^{2}}{h}+\frac{1}{2} g h^{2}\right)=-k q|q| h^{-\eta}
\end{array}\right.
$$

Similarly to the topography-only case, the smoothness assumption allows (1.12) to be rewritten under an algebraic form, as follows:

$$
-\frac{q_{0}^{2}}{\eta-1} \partial_{x} h^{\eta-1}+\frac{g}{\eta+2} \partial_{x} h^{\eta+2}+k q_{0}\left|q_{0}\right|=0 .
$$

These steady states are highly nonlinear, and exact preservation is a challenging task, see for instance $[7,53]$ and references therein. Note that, for the case of both source terms, the steady states (1.9) cannot be rewritten under an algebraic form. In addition, complex steady states also appear when studying shallow water-like systems, see for instance $[18,34,39]$ where the authors respectively deal with moving beds, temperature transport in the Ripa model, and non-hydrostatic terms. Another area of interest regarding complex steady states is the study of the Euler equations, see for instance $[23,5,24,6,62,61]$ and references therein, but this list is far from being exhaustive. 
In $[52,53]$, the authors develop a robust numerical scheme able to exactly preserve and capture the smooth steady states associated with the topography and the friction source terms. In addition, this scheme was proven to be entropy-satisfying in [12]. We now aim at providing a high-order extension in two space dimensions, while retaining the robustness property, i.e. the preservation of the water height non-negativity, and the essential wellbalancedness property. First steps have been undertaken in [11], where a well-balanced second-order MUSCL extension is proposed. Note that other work has been devoted to the development of high-order schemes which preserve the lake at rest (see for instance [17, 29]) or the moving steady states (see for instance [56, 22, 65, 21]).

In order to derive high-order reconstruction techniques, a particular attention must be paid to the preservation of the steady states. This point is easily solved when only considering the preservation of the lake at rest steady state (1.7). Indeed, it is sufficient to propose a reconstruction of the free surface, since the reconstructed water height is then obtained by solving a linear equation. The situation turns out to be very different as soon as moving steady states are considered, since we now have to deal with Bernoulli's relation (1.11). For instance, in recent works $[26,50,41]$, a direct reconstruction of this Bernoulli relation is suggested. From such a reconstruction, the water height is now solution of a nonlinear equation to be solved at each interface. This is an efficient technique but computationally costly. Here, our goal is to propose a method which only requires one evaluation of the Bernoulli relation per interface, so that we never have to solve such a nonlinear equation. This discussion highlights the computational gain, and thus the efficiency, of the designed method. Moreover, the friction source term is left mostly untreated in the high-order well-balanced literature. It turns out that our method is also able to capture friction steady states without having to solve a nonlinear equation. Therefore, our goal is to propose a well-balanced high-order strategy, valid for both friction and topography source terms, that does not rely on solving nonlinear equations, and that is applicable to the shallow water equations for two-dimensional geometries.

The paper is organized as follows. First, in section 2, after recalling the 1D well-balanced scheme following $[52,53]$, we design the 2D well-balanced scheme. Then, in section 3 , we discuss the high-order polynomial reconstruction and how to apply it to the numerical scheme. Afterwards, we detail a well-balancedness correction in section 4, designed so that the resulting scheme is both well-balanced and high-order accurate. Section 5 then presents the MOOD techniques we used to ensure the non-negativity preservation and the elimination of the spurious oscillations caused by the high-order reconstruction. Finally, section 6 is dedicated to the numerical experiments, designed to test the properties of the scheme, namely its well-balancedness, its high-order accuracy and its robustness. The simulation of the 2011 Töhoku tsunami, on real data, shows good agreement between the numerical results and the physical measurements.

\section{A well-balanced scheme in two space dimensions}

In this section, we build a two-dimensional (2D) scheme by adapting the one-dimensional (1D) well-balanced scheme from $[52,53]$. We recall the $1 \mathrm{D}$ scheme in section 2.1 for the sake of completeness, and we provide a $2 \mathrm{D}$ extension in section 2.2 .

\subsection{Reminder of the $1 D$ well-balanced scheme}

This section is devoted to recalling from $[52,53]$ the well-balanced scheme for the $1 \mathrm{D}$ shallow water equations with the source terms of topography and friction (1.8). The notation we use in this section is derived in a straightforward way from (1.4) - (1.5). The scheme has been constructed in [52, 53]; we sketch the resulting 
scheme here for the sake of completeness. First, we write the scheme as a Godunov-scheme in section 2.1.1, and we then state a semi-implicit version of the scheme in section 2.1.2.

\subsubsection{The $1 D$ scheme as a Godunov-type scheme}

Let us first recall the expression of the scheme as a Godunov-type scheme. Let $\Delta x$ be the uniform space step. We discretize the space domain $\mathbb{R}$ in cells $\left(x_{i-\frac{1}{2}}, x_{i+\frac{1}{2}}\right)$, of volume $\Delta x$ and of center $x_{i}$. We consider a piecewise constant approximate solution of (1.8) at time $t=t^{n}$, denoted by $W_{i}^{n}:=\left(h_{i}^{n}, q_{i}^{n}\right)^{\top}$ in each cell. Let the time step $\Delta t$ satisfy the following CFL condition:

$$
\Delta t \leq \frac{\Delta x}{2 \Lambda}, \text { where } \Lambda=\max _{i \in \mathbb{Z}}\left(\left|\lambda_{i+\frac{1}{2}}^{L}\right|, \lambda_{i+\frac{1}{2}}^{R}\right)
$$

with $\lambda_{L}$ and $\lambda_{R}$ some characteristic velocities, defined as follows between two states $W_{L}=\left(h_{L}, q_{L}\right)^{\top}$ and $W_{R}=$ $\left(h_{R}, q_{R}\right)^{\top}$ (see for instance [63]):

$$
\begin{aligned}
& \lambda_{L}=\min \left(-\left|u_{L}\right|-\sqrt{g h_{L}},-\left|u_{R}\right|-\sqrt{g h_{R}},-\varepsilon_{\lambda}\right) \leq-\varepsilon_{\lambda}, \\
& \lambda_{R}=\max \left(\left|u_{L}\right|+\sqrt{g h_{L}},\left|u_{R}\right|+\sqrt{g h_{R}}, \varepsilon_{\lambda}\right) \geq-\varepsilon_{\lambda}
\end{aligned}
$$

where $\varepsilon_{\lambda}$ is a positive constant that is taken equal to $10^{-10}$ in the numerical simulations. Then, the updated approximation at time $t^{n+1}=t^{n}+\Delta t$ reads:

$$
W_{i}^{n+1}=W_{i}^{n}-\frac{\Delta t}{\Delta x}\left(\lambda_{i+\frac{1}{2}}^{L}\left(W_{i+\frac{1}{2}}^{L, *}-W_{i}^{n}\right)-\lambda_{i-\frac{1}{2}}^{R}\left(W_{i-\frac{1}{2}}^{R, *}-W_{i}^{n}\right)\right) .
$$

To complete the determination of the scheme, one has to give the values of the so-called intermediate states $W_{i+\frac{1}{2}}^{L, *}$ and $W_{i-\frac{1}{2}}^{R, *}$.

Between two states $W_{L}$ and $W_{R}$, we define the intermediate heights and discharges as $W_{L}^{*}=\left(h_{L}^{*}, q_{L}^{*}\right)^{\top}$ and $W_{R}^{*}=\left(h_{R}^{*}, q_{R}^{*}\right)^{\top}$ by imposing the three properties the scheme has to satisfy:

- consistency with (1.8);

- well-balancedness;

- non-negativity preservation.

The scheme is based on the intermediate states from the well-known HLL scheme [48], whose intermediate states $\left(h_{H L L}, q_{H L L}\right)^{\top}$ are given by

$$
\begin{aligned}
& \left(\lambda_{R}-\lambda_{L}\right) h_{H L L}=\lambda_{R} h_{R}-\lambda_{L} h_{L}-[q], \\
& \left(\lambda_{R}-\lambda_{L}\right) q_{H L L}=\lambda_{R} q_{R}-\lambda_{L} q_{L}-\left[\frac{q^{2}}{h}+\frac{1}{2} g h^{2}\right] .
\end{aligned}
$$

with $[X]=X_{R}-X_{L}$ the jump of any quantity $X$. The intermediate states of the well-balanced scheme then 
read, with $X_{+}=\max (X, 0)$, by:

$$
\begin{aligned}
q^{*} & =q_{H L L}+\frac{\bar{S} \Delta x}{\lambda_{R}-\lambda_{L}}, \\
h_{L}^{*} & =\min \left(\left(h_{H L L}-\frac{\lambda_{R} \bar{S} \Delta x}{\alpha\left(\lambda_{R}-\lambda_{L}\right)}\right)_{+},\left(1-\frac{\lambda_{R}}{\lambda_{L}}\right) h_{H L L}\right), \\
h_{R}^{*} & =\min \left(\left(h_{H L L}-\frac{\lambda_{L} \bar{S} \Delta x}{\alpha\left(\lambda_{R}-\lambda_{L}\right)}\right)_{+},\left(1-\frac{\lambda_{L}}{\lambda_{R}}\right) h_{H L L}\right),
\end{aligned}
$$

where we have set

$$
\alpha=\frac{-\bar{q}^{2}}{h_{L} h_{R}}+\frac{g}{2}\left(h_{L}+h_{R}\right)
$$

with $\bar{q}$ the harmonic mean of $q_{L}$ and $q_{R}$ :

$$
\bar{q}= \begin{cases}\frac{2\left|q_{L}\right|\left|q_{R}\right|}{\left|q_{L}\right|+\left|q_{R}\right|} \operatorname{sgn}\left(q_{L}+q_{R}\right) & \text { if } q_{L} \neq 0 \text { and } q_{R} \neq 0 \\ 0 & \text { otherwise }\end{cases}
$$

In addition, the expressions (2.4) depend on a parameter $\bar{S}$, which is an approximation of the source term.

This parameter $\bar{S}$ is taken as $\bar{S}=\bar{S}^{t}+\bar{S}^{f}$, with the approximate topography source term given by:

$$
\begin{aligned}
\bar{S}^{t} \Delta x:= & \bar{S}^{t}\left(h_{L}, h_{R}, Z_{L}, Z_{R}, \Delta x\right) \Delta x \\
& = \begin{cases}\frac{g h_{R}^{2}}{2} & \text { if } q_{R}=h_{L}=0 \text { and } h_{R}+Z_{R} \leq Z_{L}, \\
-\frac{g h_{L}^{2}}{2} & \text { if } q_{L}=h_{R}=0 \text { and } h_{L}+Z_{L} \leq Z_{R}, \\
-g[Z] \frac{h_{L}+h_{R}}{2} & \text { if } h_{L}=0 \text { or } h_{R}=0, \\
-2 g[Z] \frac{h_{L} h_{R}}{h_{L}+h_{R}}+\frac{g}{2} \frac{[h]_{c}^{3}}{h_{L}+h_{R}} & \text { if } h_{L} \neq 0 \text { and } h_{R} \neq 0,\end{cases}
\end{aligned}
$$

and the approximate friction source term defined by:

$$
\bar{S}^{f}:=\bar{S}^{f}\left(h_{L}, h_{R}, q_{L}, q_{R}\right)=-k \bar{q}|\bar{q}| \overline{h^{-\eta}}
$$

with

$$
\overline{h^{-\eta}}=\frac{\left[h^{2}\right]}{2} \frac{\eta+2}{\left[h^{\eta+2}\right]}-\frac{\operatorname{sgn} \bar{q}}{k \Delta x}[h]_{c}\left(-\frac{1}{h_{L} h_{R}}+\frac{h_{L}+h_{R}}{2} \frac{\left[h^{\eta-1}\right]}{\eta-1} \frac{\eta+2}{\left[h^{\eta+2}\right]}\right) .
$$

In (2.7) and (2.9), we have introduced $[h]_{c}$ a cut-off of the jump $[h]$, required to ensure the consistency property, and given by:

$$
[h]_{c}= \begin{cases}h_{R}-h_{L} & \text { if }\left|h_{R}-h_{L}\right| \leq C \Delta x, \\ \operatorname{sgn}\left(h_{R}-h_{L}\right) C \Delta x & \text { otherwise, }\end{cases}
$$

with $C$ a positive constant that does not depend on $\Delta x$. This expression of $\bar{S}$ allows us to clarify the definition 
of well-balancedness as the preservation of steady states between $W_{L}$ and $W_{R}$, which are defined by

$$
\left\{\begin{array}{l}
{[q]=0} \\
{\left[\frac{q^{2}}{h}+\frac{1}{2} g h^{2}\right]=\bar{S}}
\end{array}\right.
$$

We also consider the following non-smooth lake at rest solutions:

$$
\left\{\begin{array} { l } 
{ q _ { 0 } = h _ { R } = 0 , } \\
{ h _ { L } + Z _ { L } \leq Z _ { R } , }
\end{array} \quad \text { or } \quad \left\{\begin{array}{l}
q_{0}=h_{L}=0 \\
h_{R}+Z_{R} \leq Z_{L}
\end{array}\right.\right.
$$

Equipped with the two parameters $\bar{S}^{t}$ and $\bar{S}^{f}$, we have fully determined the intermediate states (2.4) for $h_{L} \geq 0$ and $h_{R} \geq 0$. We thus recall the following result from [52,53], that describes the properties verified by the full one-dimensional scheme.

Theorem 1 ([52, 53]). Let $W_{i}^{n} \in \Omega$ for all $i \in \mathbb{Z}$, with $\Omega$ the admissible states space, given by (1.6). Assume that, for all $i \in \mathbb{Z}$, the intermediate states $W_{i+\frac{1}{2}}^{L, *}$ and $W_{i+\frac{1}{2}}^{R, *}$ satisfy

$$
W_{i+\frac{1}{2}}^{L, *}=\left(\begin{array}{c}
h_{L}^{*}\left(W_{i}^{n}, W_{i+1}^{n}\right) \\
q^{*}\left(W_{i}^{n}, W_{i+1}^{n}\right)
\end{array}\right) \quad \text { and } \quad W_{i+\frac{1}{2}}^{R, *}=\left(\begin{array}{c}
h_{R}^{*}\left(W_{i}^{n}, W_{i+1}^{n}\right) \\
q^{*}\left(W_{i}^{n}, W_{i+1}^{n}\right)
\end{array}\right) \text {, }
$$

where $h_{L}^{*}, h_{R}^{*}$ and $q^{*}$ are defined by (2.4). Then the Godunov-type scheme given by (2.3), under the CFL restriction (2.1), satisfies the following properties:

1. consistency with the shallow water equations with topography and friction (1.8);

2. robustness: $\forall i \in \mathbb{Z}, W_{i}^{n+1} \in \Omega$;

3. well-balancedness: if $\left(W_{i}^{n}\right)_{i \in \mathbb{Z}}$ defines a steady state according to (2.11), then $\forall i \in \mathbb{Z}, W_{i}^{n+1}=W_{i}^{n}$.

\subsubsection{Semi-implicitation of the scheme}

The scheme $(2.3)-(2.4)$ is robust, as stated by theorem 1. However, oscillations due to the stiffness of the source terms occur when simulating transitions between dry and wet areas. To address this issue, we partially follow [53] and introduce an implicit treatment of the friction source term. Let us emphasize that this implicit treatment does not involve numerically solving nonlinear equation.

Remark 1. In [53], both source terms of topography and friction receive an implicit treatment. However, because of the new definition (2.7) of $\bar{S}^{t}$, such a treatment is not possible anymore for the topography source term. Thus, the three-step scheme from [53] becomes a two-step scheme in the present paper.

We begin by rewriting the scheme $(2.3)-(2.4)$ to exhibit the numerical flux function and the numerical source terms, as follows (see [48] for instance):

$$
\left(\begin{array}{c}
h_{i}^{n+1} \\
q_{i}^{n+1}
\end{array}\right)=\left(\begin{array}{c}
h_{i}^{n} \\
q_{i}^{n}
\end{array}\right)-\frac{\Delta t}{\Delta x}\left(\mathcal{F}_{i+\frac{1}{2}}^{n}-\mathcal{F}_{i-\frac{1}{2}}^{n}\right)+\Delta t\left(\begin{array}{c}
0 \\
\left(S^{t}\right)_{i}^{n}+\left(S^{f}\right)_{i}^{n}
\end{array}\right),
$$

where $\mathcal{F}_{i+\frac{1}{2}}^{n}$ is an approximation of the physical flux at the interface $x_{i+\frac{1}{2}}$, and $\left(S^{t}\right)_{i}^{n}$ and $\left(S^{f}\right)_{i}^{n}$ are, respectively, approximations of the topography and the friction source terms within the cell $\left(x_{i-\frac{1}{2}}, x_{i+\frac{1}{2}}\right)$. The numerical 
flux $\mathcal{F}_{i+\frac{1}{2}}^{n}$ is defined by:

$$
\mathcal{F}_{i+\frac{1}{2}}^{n}:=\mathcal{F}\left(W_{i}^{n}, W_{i+1}^{n}\right)=\frac{1}{2}\left(F\left(W_{i}^{n}\right)+F\left(W_{i+1}^{n}\right)\right)+\frac{\lambda_{i+\frac{1}{2}}^{L}}{2}\left(W_{i+\frac{1}{2}}^{L, *}-W_{i}^{n}\right)+\frac{\lambda_{i+\frac{1}{2}}^{R}}{2}\left(W_{i+\frac{1}{2}}^{R, *}-W_{i+1}^{n}\right) .
$$

In addition, the numerical source terms are defined as follows:

$$
\left(S^{t}\right)_{i}^{n}=\frac{\left(\bar{S}^{t}\right)_{i-\frac{1}{2}}^{n}+\left(\bar{S}^{t}\right)_{i+\frac{1}{2}}^{n}}{2} \quad \text { and } \quad\left(S^{f}\right)_{i}^{n}=\frac{\left(\bar{S}^{f}\right)_{i-\frac{1}{2}}^{n}+\left(\bar{S}^{f}\right)_{i+\frac{1}{2}}^{n}}{2}
$$

where $\left(\bar{S}^{t}\right)_{i+\frac{1}{2}}^{n}$ and $\left(\bar{S}^{f}\right)_{i+\frac{1}{2}}^{n}$ are given with clear notation by:

$$
\begin{aligned}
& \left(\bar{S}^{t}\right)_{i+\frac{1}{2}}^{n}=\bar{S}^{t}\left(h_{i}^{n}, h_{i+1}^{n}, q_{i}^{n}, q_{i+1}^{n}, Z_{i}, Z_{i+1}, \Delta x\right), \\
& \left(\bar{S}^{f}\right)_{i+\frac{1}{2}}^{n}=\bar{S}^{f}\left(h_{i}^{n}, h_{i+1}^{n}, q_{i}^{n}, q_{i+1}^{n}\right),
\end{aligned}
$$

with the functions $\bar{S}^{t}$ and $\bar{S}^{f}$ given by (2.7) and (2.8).

We now introduce a semi-implicit strategy, based on a splitting method (see [15, 63] for more details), which involves an explicit treatment of the hyperbolic part and of the topography source term, and an implicit treatment of the friction source term. Two computational steps are therefore necessary. The first step is devoted to the explicit treatment of the flux and the topography source term, namely:

$$
\left(\begin{array}{c}
h_{i}^{n+\frac{1}{2}} \\
q_{i}^{n+\frac{1}{2}}
\end{array}\right)=\left(\begin{array}{c}
h_{i}^{n} \\
q_{i}^{n}
\end{array}\right)-\frac{\Delta t}{\Delta x}\left(\mathcal{F}_{i+\frac{1}{2}}^{n}-\mathcal{F}_{i-\frac{1}{2}}^{n}\right)+\Delta t\left(\begin{array}{c}
0 \\
\left(S^{t}\right)_{i}^{n}
\end{array}\right) .
$$

The second step concerns the friction contribution, and we get:

$$
\left\{\begin{array}{l}
h_{i}^{n+1}=h_{i}^{n+\frac{1}{2}} \\
q_{i}^{n+1}=\frac{\left(\overline{h^{\eta}}\right)_{i}^{n+1} q_{i}^{n+\frac{1}{2}}}{\left(\overline{h^{\eta}}\right)_{i}^{n+1}+k \Delta t\left|q_{i}^{n+\frac{1}{2}}\right|},
\end{array}\right.
$$

where $\left(\overline{h^{\eta}}\right)_{i}^{n+1}$ is a consistent average of $h_{i-1}^{n+1}, h_{i}^{n+1}$ and $h_{i+1}^{n+1}$ designed to recover the well-balancedness property. More details on the process followed to obtain a relevant average $\left(\overline{h^{\eta}}\right)_{i}^{n+1}$ are present in [53]. The quantity $\left(\overline{h^{\eta}}\right)_{i}^{n+1}$ is defined by:

$$
\left(\overline{h^{\eta}}\right)_{i}^{n+1}=\frac{2 k \mu_{i}^{n+\frac{1}{2}} \Delta x}{k \mu_{i}^{n} \Delta x\left(\beta_{i-\frac{1}{2}}^{n+1}+\beta_{i+\frac{1}{2}}^{n+1}\right)-\left(\gamma_{i-\frac{1}{2}}^{n+1}+\gamma_{i+\frac{1}{2}}^{n+1}\right)}+k \Delta t \mu_{i}^{n+\frac{1}{2}} q_{i}^{n},
$$

with $\mu_{i}^{n}=\operatorname{sgn}\left(q_{i}^{n}\right)$ and $\mu_{i}^{n+\frac{1}{2}}=\operatorname{sgn}\left(q_{i}^{n+\frac{1}{2}}\right)$, and where $\beta_{i+\frac{1}{2}}^{n+1}$ and $\gamma_{i+\frac{1}{2}}^{n+1}$ are given by:

$$
\begin{aligned}
\beta_{i+\frac{1}{2}}^{n+1} & =\frac{\eta+2}{2} \frac{\left(h_{i+1}^{n+1}\right)^{2}-\left(h_{i}^{n+1}\right)^{2}}{\left(h_{i+1}^{n+1}\right)^{\eta+2}-\left(h_{i}^{n+1}\right)^{\eta+2}}, \\
\gamma_{i+\frac{1}{2}}^{n+1} & =\frac{1}{h_{i+1}^{n+1}}-\frac{1}{h_{i}^{n+1}}+\beta_{i+\frac{1}{2}}^{n+1} \frac{\left(h_{i+1}^{n+1}\right)^{\eta-1}-\left(h_{i}^{n+1}\right)^{\eta-1}}{\eta-1} .
\end{aligned}
$$

Equipped with this two-step scheme, we can state the following result. 
Theorem 2. The two-step scheme (2.15) - (2.16) is consistent with the shallow water equations (1.8), robust, and well-balanced.

A similar result has been proven in [53] for the three-step scheme used in that paper. The proof of this result for the current two-step scheme uses the same ingredients, and we do not present it here for the sake of conciseness.

\subsection{A two-dimensional extension}

We now propose a two-dimensional extension on a Cartesian grid of the scheme presented in the previous subsection, in order to approximate solutions of (1.1). First, we introduce the notation we use regarding the discretization of the space domain $\mathbb{R}^{2}$. Then, we present the $2 \mathrm{D}$ scheme as a convex combination of $1 \mathrm{D}$ schemes.

\subsubsection{Space discretization}

The discretization of the space domain $\mathbb{R}^{2}$ consists in a Cartesian mesh of uniform cells, defined by:

$$
c_{i, j}=\left(x_{i, j}-\frac{\Delta x}{2}, x_{i, j}+\frac{\Delta x}{2}\right) \times\left(y_{i, j}-\frac{\Delta y}{2}, y_{i, j}+\frac{\Delta y}{2}\right)
$$

where $\left(x_{i, j}, y_{i, j}\right)$ is the cell center of $c_{i, j}$. We denote by $\left|c_{i, j}\right|=\Delta x \Delta y$ the area of the cell $c_{i, j}$. The piecewise constant approximate solution, within the cell $c_{i, j}$ and at time $t^{n}$, is denoted by $W_{i, j}^{n}$.

\subsubsection{Construction of the a $2 D$ scheme}

We build the two-dimensional extension as a convex combination of one-dimensional schemes. The reader is referred for instance to $[57,10,14,13]$, where such a convex combination is presented for an unstructured mesh. In our particular case of a Cartesian mesh, the first step of the two-step scheme reads as follows:

$$
W_{i, j}^{n+\frac{1}{2}}=\frac{1}{4}\left(W_{i+\frac{1}{2}, j}^{n+\frac{1}{2}}+W_{i-\frac{1}{2}, j}^{n+\frac{1}{2}}+W_{i, j+\frac{1}{2}}^{n+\frac{1}{2}}+W_{i, j-\frac{1}{2}}^{n+\frac{1}{2}}\right)
$$

with the notation

$$
\begin{aligned}
& W_{i-\frac{1}{2}, j}^{n+\frac{1}{2}}=W_{i, j}^{n}-\frac{4 \Delta t}{\Delta x}\left(\mathcal{F}\left(W_{i, j}^{n}, W_{i, j}^{n}\right)-\mathcal{F}\left(W_{i, j}^{n}, W_{i-1, j}^{n}\right)\right)+2 \Delta t\left(0,\left(\bar{S}_{x}^{t}\right)_{i-\frac{1}{2}, j}^{n}, 0\right)^{\top}, \\
& W_{i+\frac{1}{2}, j}^{n+\frac{1}{2}}=W_{i, j}^{n}-\frac{4 \Delta t}{\Delta x}\left(\mathcal{F}\left(W_{i, j}^{n}, W_{i+1, j}^{n}\right)-\mathcal{F}\left(W_{i, j}^{n}, W_{i, j}^{n}\right)\right)+2 \Delta t\left(0,\left(\bar{S}_{x}^{t}\right)_{i+\frac{1}{2}, j}^{n}, 0\right)^{\top}, \\
& W_{i, j-\frac{1}{2}}^{n+\frac{1}{2}}=W_{i, j}^{n}-\frac{4 \Delta t}{\Delta y}\left(\mathcal{G}\left(W_{i, j}^{n}, W_{i, j}^{n}\right)-\mathcal{G}\left(W_{i, j}^{n}, W_{i, j-1}^{n}\right)\right)+2 \Delta t\left(0,0,\left(\bar{S}_{y}^{t}\right)_{i, j-\frac{1}{2}}^{n}\right)^{\top}, \\
& W_{i, j+\frac{1}{2}}^{n+\frac{1}{2}}=W_{i, j}^{n}-\frac{4 \Delta t}{\Delta y}\left(\mathcal{G}\left(W_{i, j}^{n}, W_{i, j+1}^{n}\right)-\mathcal{G}\left(W_{i, j}^{n}, W_{i, j}^{n}\right)\right)+2 \Delta t\left(0,0,\left(\bar{S}_{y}^{t}\right)_{i, j+\frac{1}{2}}^{n}\right)^{\top},
\end{aligned}
$$

where $\left(\bar{S}_{x}^{t}\right)_{i+\frac{1}{2}, j}^{n}$ and $\left(\bar{S}_{y}^{t}\right)_{i, j+\frac{1}{2}}^{n}$ are defined by:

$$
\begin{aligned}
& \left(\bar{S}_{x}^{t}\right)_{i+\frac{1}{2}, j}^{n}=\bar{S}^{t}\left(h_{i, j}^{n}, h_{i+1, j}^{n},\left(q_{x}\right)_{i, j}^{n},\left(q_{x}\right)_{i+1, j}^{n}, Z_{i, j}, Z_{i+1, j}, \Delta x\right), \\
& \left(\bar{S}_{y}^{t}\right)_{i, j+\frac{1}{2}}^{n}=\bar{S}^{t}\left(h_{i, j}^{n}, h_{i, j+1}^{n},\left(q_{y}\right)_{i, j}^{n},\left(q_{y}\right)_{i, j+1}^{n}, Z_{i, j}, Z_{i, j+1}, \Delta y\right),
\end{aligned}
$$

with $\bar{S}^{t}$ defined by (2.7). Note that (2.20) represents a collection of four one-dimensional schemes, and that (2.19) is nothing but a convex combination of these schemes. 
In (2.20), the numerical flux function $\mathcal{F}$ is the $1 \mathrm{D}$ function in the $x$-direction defined in (2.13) and the numerical flux in the $y$-direction, $\mathcal{G}$, derives from $\mathcal{F}$ by using classical rotational invariance properties (see for instance [40]). The equation (2.19) can be rewritten using (2.20) under the following classical form:

$$
\begin{aligned}
W_{i, j}^{n+\frac{1}{2}}=W_{i, j}^{n} & -\frac{\Delta t}{\Delta x}\left(\mathcal{F}\left(W_{i, j}^{n}, W_{i+1, j}^{n}\right)-\mathcal{F}\left(W_{i-1, j}^{n}, W_{i, j}^{n}\right)\right) \\
& -\frac{\Delta t}{\Delta y}\left(\mathcal{G}\left(W_{i, j}^{n}, W_{i, j+1}^{n}\right)-\mathcal{G}\left(W_{i, j-1}^{n}, W_{i, j}^{n}\right)\right) \\
& +\Delta t\left(\begin{array}{c}
0 \\
\left(\boldsymbol{S}^{t}\right)_{i, j}^{n}
\end{array}\right),
\end{aligned}
$$

together with

$$
\left(\boldsymbol{S}^{t}\right)_{i, j}^{n}=\frac{1}{2}\left(\begin{array}{c}
\left(\bar{S}_{x}^{t}\right)_{i-\frac{1}{2}, j}^{n}+\left(\bar{S}_{x}^{t}\right)_{i+\frac{1}{2}, j}^{n} \\
\left(\bar{S}_{y}^{t}\right)_{i, j-\frac{1}{2}}^{n}+\left(\bar{S}_{y}^{t}\right)_{i, j+\frac{1}{2}}^{n}
\end{array}\right) .
$$

The time step is chosen to satisfy the following CFL condition:

$$
\Delta t=\frac{\delta}{2 \Lambda}
$$

where $\delta=\min (\Delta x, \Delta y)$ and $\Lambda$ stands for the maximum of all characteristic velocities at each interface.

To build the second step of the scheme, we address the implicitation of the friction contribution. Similarly to the $1 \mathrm{D}$ case, we solve the following initial value problem:

$$
\left\{\begin{array} { l } 
{ h ^ { \prime } = 0 , } \\
{ \boldsymbol { q } ^ { \prime } = - k \boldsymbol { q } \| \boldsymbol { q } \| h ^ { - \eta } , }
\end{array} \text { with initial data } \left\{\begin{array}{l}
h(0)=h_{i, j}^{n+\frac{1}{2}} \\
\boldsymbol{q}(0)=\boldsymbol{q}_{i, j}^{n+\frac{1}{2}}
\end{array}\right.\right.
$$

This initial value problem again admits an analytic solution, given for $t \in[0, \Delta t]$ by:

$$
\left\{\begin{array}{l}
h(t)=h(0), \\
\boldsymbol{q}(t)=\frac{h(0)^{\eta} \boldsymbol{q}(0)}{h(0)^{\eta}+k t\|\boldsymbol{q}(0)\|} .
\end{array}\right.
$$

We slightly modify the expression of the discharge given by (2.23) to recover the well-balancedness property, and, the updated state reads, with $\boldsymbol{q}=\left(q_{x}, q_{y}\right)^{\mathrm{T}}$ :

$$
\left\{\begin{array}{l}
h_{i, j}^{n+1}=h_{i, j}^{n+\frac{1}{2}} \\
\left(q_{x}\right)_{i, j}^{n+1}=\frac{\left(\overline{h_{x}^{\eta}}\right)_{i, j}^{n+1}\left(q_{x}\right)_{i, j}^{n+\frac{1}{2}}}{\left(\overline{h_{x}^{\eta}}\right)_{i, j}^{n+1}+k \Delta t\left\|\boldsymbol{q}_{i, j}^{n+\frac{1}{2}}\right\|}, \\
\left(q_{y}\right)_{i, j}^{n+1}=\frac{\left(\overline{h_{y}^{\eta}}\right)_{i, j}^{n+1}\left(q_{y}\right)_{i, j}^{n+\frac{1}{2}}}{\left(\overline{h_{y}^{\eta}}\right)_{i, j}^{n+1}+k \Delta t\left\|\boldsymbol{q}_{i, j}^{n+\frac{1}{2}}\right\|},
\end{array}\right.
$$

with $\left(\overline{h_{x}^{\eta}}\right)_{i, j}^{n+1}$ and $\left(\overline{h_{y}^{\eta}}\right)_{i, j}^{n+1}$ given by $(2.17)$ in the $x$ - and $y$-direction, respectively.

The 2D scheme is now complete. To state its properties, we require the following definition. 
Definition. The vector $\left(W_{i, j}^{n}\right)_{(i, j) \in \mathbb{Z}^{2}}$ is said to define a steady state in the $x$-direction if:

- $\forall(i, j) \in \mathbb{Z}^{2}, W_{i, j+1}^{n}=W_{i, j}^{n}$;

- $\forall(i, j) \in \mathbb{Z}^{2},\left(q_{y}\right)_{i, j}^{n}=0$;

- $\forall(i, j) \in \mathbb{Z}^{2}$, the pairs $\left(h_{i, j}^{n},\left(q_{x}\right)_{i, j}^{n}, Z_{i, j}\right)^{\top}$ and $\left(h_{i+1, j}^{n},\left(q_{x}\right)_{i+1, j}^{n}, Z_{i+1, j}\right)^{\top}$ satisfy $(2.11)$ or $(2.12)$.

Similarly, $\left(W_{i, j}^{n}\right)_{(i, j) \in \mathbb{Z}^{2}}$ is said to define a steady state in the $y$-direction if:

- $\forall(i, j) \in \mathbb{Z}^{2}, W_{i+1, j}^{n}=W_{i, j}^{n}$;

- $\forall(i, j) \in \mathbb{Z}^{2},\left(q_{x}\right)_{i, j}^{n}=0$;

- $\forall(i, j) \in \mathbb{Z}^{2}$, the pairs $\left(h_{i, j}^{n},\left(q_{y}\right)_{i, j}^{n}, Z_{i, j}\right)^{\top}$ and $\left(h_{i, j+1}^{n},\left(q_{y}\right)_{i, j+1}^{n}, Z_{i, j+1}\right)^{\top}$ satisfy $(2.11)$ or $(2.12)$.

The following result is then satisfied by the $2 \mathrm{D}$ scheme:

Theorem 3. Under the CFL condition (2.22), the two-dimensional two-step scheme (2.21) - (2.24) is robust and well-balanced in the $x$ - and $y$-directions:

1. Robustness: if $W_{i, j}^{n} \in \Omega$ for all $(i, j) \in \mathbb{Z}^{2}$, then $W_{i, j}^{n+1} \in \Omega$ for all $(i, j) \in \mathbb{Z}^{2}$.

2. Well-balancedness in the $x$ - and $y$-directions: if $\left(W_{i, j}^{n}\right)_{(i, j) \in \mathbb{Z}^{2}}$ defines a steady state in the $x$ - or the $y$ direction, then for all $(i, j) \in \mathbb{Z}^{2}, W_{i, j}^{n+1}=W_{i, j}^{n}$.

Proof. The proof of this theorem revolves rewriting the first step (2.21) of the 2D scheme under the form of a convex combination of 1D schemes, given by (2.19) and (2.20). Then, each 1D scheme defined by (2.20) enjoys the same properties as the truly 1D scheme (2.15). In addition, these properties are satisfied by the convex combination (2.19). Therefore, the robustness of the two-step scheme is immediate, since the 1D schemes are robust.

In order to establish the well-balancedness, assume that $\left(W_{i, j}^{n}\right)_{(i, j) \in \mathbb{Z}^{2}}$ defines a steady state in the $x$-direction. Therefore, the sum of the vertical fluxes in (2.21) and the $y$ contribution of the topography vanishes. Thus, the first step of the scheme turns out to be the 1D first step following the $x$-direction. Then, the $y$ contribution of the friction source term vanishes, leaving only the $x$ contribution, which is the same as in the $1 \mathrm{D}$ case. Therefore, theorem 2 applies, and $W_{i, j}^{n+1}=W_{i, j}^{n}$ for all $(i, j) \in \mathbb{Z}^{2}$. A similar chain of arguments can be applied to prove the preservation of the steady states in the $y$-direction.

Remark 2. Note that the lake at rest steady state (1.7) is a particular case of the steady states defined in the $x$ and $y$-directions. Indeed, taking a steady state in the $x$-direction with $q_{x}=0$ yields the lake at rest. Similarly, the lake at rest is obtained by taking a steady state in the $y$-direction with $q_{y}=0$. Therefore, from theorem 3 , any two-dimensional lake at rest steady state given by (1.7) or by (2.12) is exactly preserved by the $2 \mathrm{D}$ scheme.

Remark 3. The scheme we have built preserves the 1D moving steady states, in addition to the 2D steady states at rest. The case of the fully $2 \mathrm{D}$ steady states, where the discharge is divergence-free instead of being merely constant, is a much more arduous task, and lies outside the scope of this manuscript. 


\section{High-order strategy}

Equipped with the first-order 2D scheme built in the previous section, we now build a high-order 2D scheme. First, we focus on the reconstruction strategy used to obtain reconstructed variables. Next, we present the high-order scheme that takes advantage of these reconstructed variables.

From now on and until the end of the paper, the degree of the polynomial reconstruction is denoted by $d \geq 0$. Moreover, for the sake of simplicity, we denote the reconstructed variables by $\varphi \in\left\{h, q_{x}, q_{y}, h+Z\right\}$. Note that a polynomial reconstruction of degree $d$ provides a scheme of order $(d+1)$.

\subsection{Obtaining reconstructed variables}

We begin by presenting the polynomial reconstruction strategy, introduced in [28, 35] (see also [36, 29] for more details). In the aforementioned papers, a reconstruction of the variable $\varphi$ within the cell $c_{i, j}$ is provided. In the remainder of this subsection, we apply this polynomial reconstruction procedure to our specific case of a uniform Cartesian mesh. The reconstructed variables are thus defined as follows:

$$
\hat{\varphi}_{i, j}(x, y ; d)=\varphi_{i, j}+\sum_{|\alpha| \in \llbracket 1, d \rrbracket} R_{i, j}^{\alpha}\left(\left(x-x_{i, j}\right)^{\alpha_{1}}\left(y-y_{i, j}\right)^{\alpha_{2}}-M_{i, j}^{\alpha}\right),
$$

where $\alpha=\left(\alpha_{1}, \alpha_{2}\right) \in \mathbb{N}^{2}$ is a multi-index, $|\alpha|=\alpha_{1}+\alpha_{2}$ is its length, and $\left(R_{i, j}^{\alpha}\right)_{|\alpha| \in \llbracket 1, d \rrbracket}$ are the polynomial coefficients.

The quantity $M_{i, j}^{\alpha}$ is introduced in (3.1) to ensure that the following conservation property holds for the polynomial $\hat{\varphi}_{i, j}(x, y ; d)$ :

$$
\frac{1}{\left|c_{i, j}\right|} \int_{c_{i, j}} \hat{\varphi}_{i, j}(x, y ; d) d x d y=\varphi_{i, j}
$$

Thus, we define $M_{i, j}^{\alpha}$ as follows:

$$
M_{i, j}^{\alpha}=\frac{1}{\left|c_{i, j}\right|} \int_{c_{i, j}}\left(x-x_{i, j}\right)^{\alpha_{1}}\left(y-y_{i, j}\right)^{\alpha_{2}} d x d y
$$

Using the definition (2.18) of the cell $c_{i, j}$, we exactly compute the above integral, to get:

$$
M^{\alpha}=\frac{1+(-1)^{\alpha_{1}}}{2\left(\alpha_{1}+1\right)}\left(\frac{\Delta x}{2}\right)^{\alpha_{1}} \frac{1+(-1)^{\alpha_{2}}}{2\left(\alpha_{2}+1\right)}\left(\frac{\Delta y}{2}\right)^{\alpha_{2}}
$$

where we have dropped the subscript for the sake of clarity, since $M_{i, j}^{\alpha}$ does not actually depend on the cell $c_{i, j}$ in the uniform Cartesian situation.

Let $\Sigma_{i, j}^{d}$ be the stencil made of cells neighboring $c_{i, j}$ for a reconstruction of degree $d$. The stencil only depends on the degree of the polynomial reconstruction, and its construction will be detailed later on. The weights are chosen so as to minimize, in a least squares sense, the error between the average of the polynomial and the approximate solution on the cells of $\Sigma_{i, j}^{d}$. The polynomial coefficients $R_{i, j}^{\alpha}$ are therefore determined to minimize the following quadratic functional (see [28]):

$$
E_{i, j}\left(R_{i, j}\right)=\sum_{l \in \Sigma_{i, j}^{d}}\left(\frac{1}{\left|c_{l}\right|} \int_{c_{l}} \hat{\varphi}_{i, j}(x, y ; d) d x d y-\varphi_{l}\right)^{2}
$$


where $R_{i, j}=\left(R_{i, j}^{\alpha}\right)_{|\alpha| \in \llbracket 1, d \rrbracket}$. After [28] (see also [60] for more details), we rewrite below the minimization problem (3.2) as a linear system, whose solution minimizes $E_{i, j}$.

After integrating (3.1) over the cell $c_{l} \in \Sigma_{i, j}^{d}$ and performing straightforward computations, we obtain

$$
\begin{aligned}
& \frac{1}{\left|c_{l}\right|} \int_{c_{l}} \hat{\varphi}_{i, j}(x, y ; d) d x d y=\varphi_{i, j}+ \\
& \quad \sum_{|\alpha| \in \llbracket 1, d \rrbracket} R_{i, j}^{\alpha}\left(\frac{1}{\left|c_{l}\right|}\left(\int_{x_{l}-x_{i, j}-\Delta x / 2}^{x_{l}-x_{i, j}+\Delta x / 2} \chi_{i, j}^{\alpha_{1}} d \chi_{i, j}\right)\left(\int_{y_{l}-y_{i, j}-\Delta y / 2}^{y_{l}-y_{i, j}+\Delta y / 2} v_{i, j}^{\alpha_{2}} d v_{i, j}\right)-M^{\alpha}\right),
\end{aligned}
$$

where $\left(x_{l}, y_{l}\right)$ is the center of the cell $c_{l}$, and where we have introduced the change of variables $\left(\chi_{i, j}, v_{i, j}\right)=$ $\left(x-x_{i, j}, y-y_{i, j}\right)$. Note that there exist $\left(\sigma_{x}^{l}, \sigma_{y}^{l}\right) \in \mathbb{Z}^{2}$ such that $x_{l}-x_{i, j}=\sigma_{x}^{l} \Delta x$ and $y_{l}-y_{i, j}=\sigma_{y}^{l} \Delta y$. The pair of integers $\left(\sigma_{x}^{l}, \sigma_{y}^{l}\right)$ represent the position of the cell $c_{l}$ relatively to the position of $c_{i, j}$, as shown in figure 1 . Note that $\left(\sigma_{x}^{l}, \sigma_{y}^{l}\right)$ does not depend on the absolute position of the cell $c_{i, j}$, since our Cartesian mesh is uniform and the stencil size is the same for each cell.

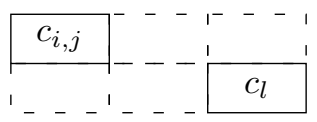

Figure 1: Relative position of the cell $c_{l}$ with respect to $c_{i, j}$. In this example, $\sigma_{x}^{l}=2$ and $\sigma_{y}^{l}=-1$.

Using $\sigma_{x}^{l}$ and $\sigma_{y}^{l}$ in (3.3), we get:

$$
\begin{aligned}
& \frac{1}{\Delta x} \int_{x_{l}-x_{i, j}-\Delta x / 2}^{x_{l}-x_{i, j}+\Delta x / 2} \chi_{i, j}^{\alpha_{1}} d \chi_{i, j}=\frac{\left(2 \sigma_{x}^{l}+1\right)^{\alpha_{1}+1}-\left(2 \sigma_{x}^{l}-1\right)^{\alpha_{1}+1}}{2\left(\alpha_{1}+1\right)}\left(\frac{\Delta x}{2}\right)^{\alpha_{1}}, \\
& \frac{1}{\Delta y} \int_{y_{l}-y_{i, j}-\Delta y / 2}^{y_{l}-y_{i, j}+\Delta y / 2} v_{i, j}^{\alpha_{2}} d v_{i, j}=\frac{\left(2 \sigma_{y}^{l}+1\right)^{\alpha_{2}+1}-\left(2 \sigma_{y}^{l}-1\right)^{\alpha_{2}+1}}{2\left(\alpha_{2}+1\right)}\left(\frac{\Delta y}{2}\right)^{\alpha_{2}} .
\end{aligned}
$$

Thus, from (3.3), we obtain

$$
\begin{gathered}
\frac{1}{\left|c_{l}\right|} \int_{c_{l}} \hat{\varphi}_{i, j}(x, y ; d) d x d y=\varphi_{i, j}+ \\
\sum_{|\alpha| \in \llbracket 1, d \rrbracket} R_{i, j}^{\alpha}\left(\frac{\left(2 \sigma_{x}^{l}+1\right)^{\alpha_{1}+1}-\left(2 \sigma_{x}^{l}-1\right)^{\alpha_{1}+1}}{2\left(\alpha_{1}+1\right)}\left(\frac{\Delta x}{2}\right)^{\alpha_{1}} \frac{\left(2 \sigma_{y}^{l}+1\right)^{\alpha_{2}+1}-\left(2 \sigma_{y}^{l}-1\right)^{\alpha_{2}+1}}{2\left(\alpha_{2}+1\right)}\left(\frac{\Delta y}{2}\right)^{\alpha_{2}}-M^{\alpha}\right) .
\end{gathered}
$$

Therefore, plugging (3.4) into (3.2), we have $E_{i, j}\left(R_{i, j}\right)=\left\|X R_{i, j}-\Phi_{i, j}\right\|^{2}$, where:

- $R_{i, j}=\left(R_{i, j}^{\alpha}\right)_{|\alpha| \in \llbracket 1, d \rrbracket}$ is the unknown vector;

- $\Phi_{i, j}=\left(\varphi_{l}-\varphi_{i, j}\right)_{l \in \Sigma_{i, j}^{d}}$;

- the matrix $X$ is defined as follows:

$$
\begin{aligned}
X= & {\left[\frac{\left(2 \sigma_{x}^{l}+1\right)^{\alpha_{1}+1}-\left(2 \sigma_{x}^{l}-1\right)^{\alpha_{1}+1}}{2\left(\alpha_{1}+1\right)}\left(\frac{\Delta x}{2}\right)^{\alpha_{1}} .\right.} \\
& \left.\frac{\left(2 \sigma_{y}^{l}+1\right)^{\alpha_{2}+1}-\left(2 \sigma_{y}^{l}-1\right)^{\alpha_{2}+1}}{2\left(\alpha_{2}+1\right)}\left(\frac{\Delta y}{2}\right)^{\alpha_{2}}-M^{\alpha}\right]_{l \in \Sigma_{i, j}^{d},|\alpha| \in \llbracket 1, d \rrbracket} .
\end{aligned}
$$


Finally, we obtain $R_{i, j}$ by using the normal equation associated to the minimization problem, as follows:

$$
X^{\top} X R_{i, j}=X^{\top} \Phi_{i, j}
$$

Remark 4. The reader is referred to $[28,35]$ for more details on how to efficiently solve the linear system (3.5). Indeed, since $X$ only depends on the fixed geometry, we avoid solving the linear system at each time iteration thanks to the Moore-Penrose pseudoinverse of $X$. More details on this pseudoinverse can be found in [60].

Remark 5. In order to ensure that there exists a solution to the minimization problem (3.2), we need more information from the stencil than we have reconstruction weights. Thus, we need $\# \Sigma_{i, j}^{d}>\#\left\{\alpha \in \mathbb{N}^{2} ;|\alpha| \in\right.$ $\llbracket 1, d \rrbracket\}$. After straightforward computations, we have the following lower bound on the size of the stencil:

$$
\# \Sigma_{i, j}^{d}>\frac{(d+1)(d+2)}{2}-1
$$

Therefore, to determine the stencil, we take the smallest symmetric stencil whose size satisfies (3.6) and that leads to the matrix $X$ being invertible. These choices are detailed in figure 2, for $d \in \llbracket 1,5 \rrbracket$.
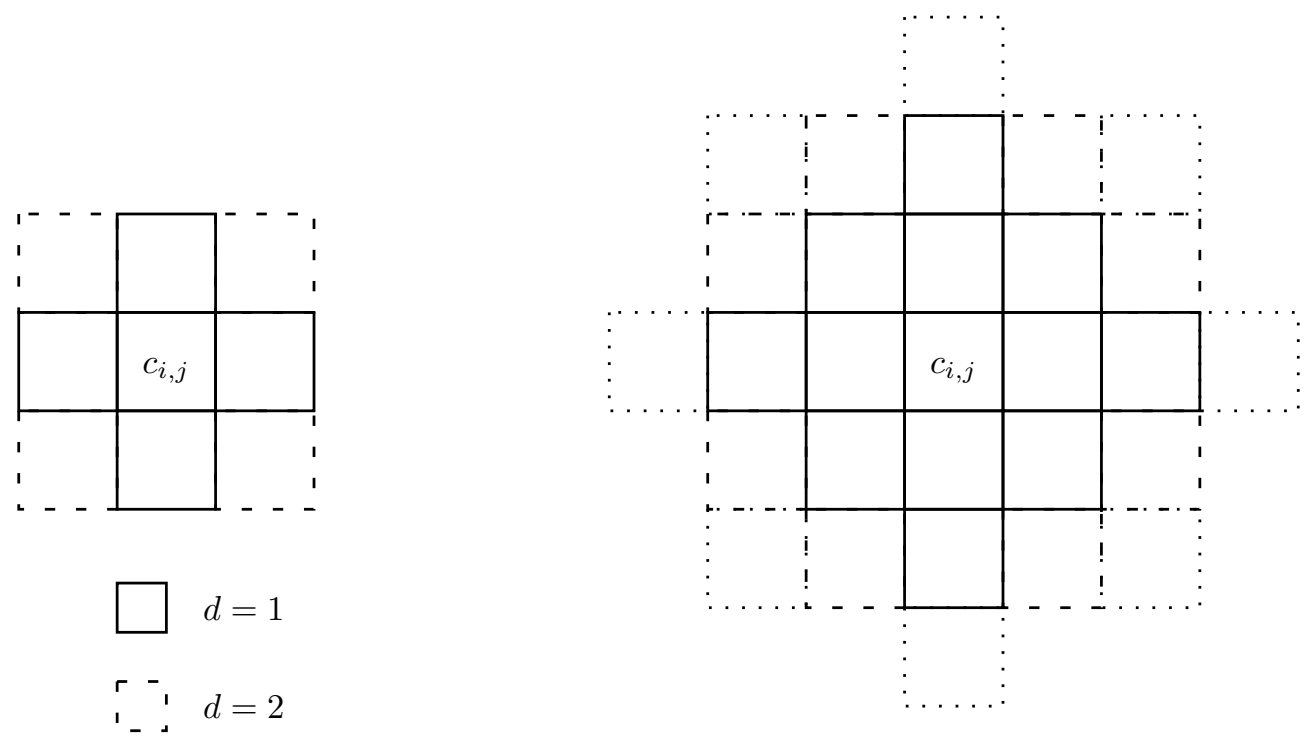

$$
\begin{array}{ll}
\square & d=3 \\
r- & d=4 \\
\hdashline & d=4 \\
\vdots \ldots & d=5
\end{array}
$$

Figure 2: Representation of the stencil $\Sigma_{i, j}^{d}$ for $d \in \llbracket 1,5 \rrbracket$. The lower order stencils are always included in the higher order ones. For the sake of simplicity, we take $\Delta x=\Delta y$ in this figure.

\subsection{The high-order scheme}

Equipped with the polynomial reconstruction, our goal is now to obtain a high-order scheme, but we face a new difficulty since this high-order scheme will not be well-balanced due to the polynomial reconstruction. In a 
later section, we shall introduce a correction to ensure the well-balancedness of the high-order scheme. We first present the high-order procedure in space, then its associated high-order time discretization.

\subsubsection{High-order space discretization}

In order to improve the spatial order of accuracy of the scheme, we numerically integrate the flux at the interfaces, which requires high-order quadrature formulas based on Gauss points. The number of Gauss points $N_{G}$ depends only on the degree $d$, and is given by

$$
N_{G}=1+\left\lfloor\frac{d}{2}\right\rfloor
$$

Let $e_{i+\frac{1}{2}, j}$ be the common interface between cells $c_{i, j}$ and $c_{i+1, j}$. The $r^{\text {th }}$ Gauss point on $e_{i+\frac{1}{2}, j}$ is denoted by $\zeta_{i+\frac{1}{2}, j}^{r}$, with the associated weight $\xi_{r}$ (see for instance [1] for more details on the coordinates of the Gauss points as well as their weights). Figure 3 shows the approximate location of the Gauss points on the edges of cell $c_{i, j}$ in the specific case where $N_{G}=2$.

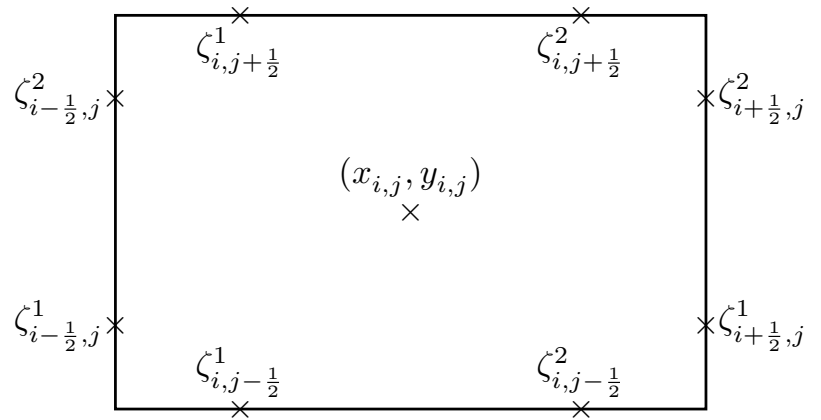

Figure 3: Approximate location of the Gauss points on the edges of cell $c_{i, j}$. We have assumed $N_{G}=2$ for this figure.

The high-order scheme reads (see [19, 35, 29] for instance):

$$
\begin{aligned}
W_{i, j}^{n+1}=W_{i, j}^{n} & -\sum_{r=1}^{N_{G}} \xi_{r}\left[\frac{\Delta t}{\Delta x}\left(\mathcal{F}_{i+\frac{1}{2}, j, r}^{n}-\mathcal{F}_{i-\frac{1}{2}, j, r}^{n}\right)\right] \\
& -\sum_{r=1}^{N_{G}} \xi_{r}\left[\frac{\Delta t}{\Delta y}\left(\mathcal{G}_{i, j+\frac{1}{2}, r}^{n}-\mathcal{G}_{i, j-\frac{1}{2}, r}^{n}\right)\right]+\Delta t\left(\mathcal{S}^{t}\right)_{i, j}^{n}+\Delta t\left(\mathcal{S}^{f}\right)_{i, j}^{n} .
\end{aligned}
$$

The quantities $\mathcal{F}_{i+\frac{1}{2}, j, r}^{n}$ and $\mathcal{G}_{i, j+\frac{1}{2}, r}^{n}$ are the numerical fluxes evaluated at the edge Gauss points, given as follows:

$$
\begin{aligned}
\mathcal{F}_{i+\frac{1}{2}, j, r}^{n} & =\mathcal{F}\left(\widehat{W}_{i, j}^{n}\left(\zeta_{i+\frac{1}{2}, j}^{r} ; d\right), \widehat{W}_{i+1, j}^{n}\left(\zeta_{i+\frac{1}{2}, j}^{r} ; d\right)\right), \\
\mathcal{G}_{i, j+\frac{1}{2}, r}^{n} & =\mathcal{G}\left(\widehat{W}_{i, j}^{n}\left(\zeta_{i, j+\frac{1}{2}}^{r} ; d\right), \widehat{W}_{i, j+1}^{n}\left(\zeta_{i, j+\frac{1}{2}}^{r} ; d\right)\right) .
\end{aligned}
$$

In (3.8), $\widehat{W}_{i, j}^{n}$ is the polynomial function containing the polynomial reconstructions of $h, q_{x}$ and $q_{y}$ within the cell $c_{i, j}$, and the functions $\mathcal{F}$ and $\mathcal{G}$ are the same as in (2.20), with the notable exception of the approximate friction source term $\bar{S}^{f}$ within the numerical flux, which is no longer defined by (2.8). Indeed, so as not to introduce an error of the order of $\Delta x$ in the high-order flux approximation, we have replaced the definition (2.8) of $\bar{S}^{f}$ with 
the following expression:

$$
\bar{S}^{f}:=\bar{S}^{f}\left(h_{L}, h_{R}, q_{L}, q_{R}\right)=-k \bar{q}|\bar{q}| \overline{h^{-\eta}} \Delta x^{d},
$$

where $\bar{q}$ is defined by $(2.6)$ and $\overline{h^{-\eta}}$ is given by:

$$
\overline{h^{-\eta}}=\frac{\left[h^{2}\right]}{2} \frac{\eta+2}{\left[h^{\eta+2}\right]}-\frac{\bar{\mu}}{k \Delta x^{d+1}}[h]_{c}\left(-\frac{1}{h_{L} h_{R}}+\frac{h_{L}+h_{R}}{2} \frac{\left[h^{\eta-1}\right]}{\eta-1} \frac{\eta+2}{\left[h^{\eta+2}\right]}\right)
$$

instead of (2.9). Note that, if $d=0$, the expressions (3.9) and (3.10) coincide with (2.8) and (2.9), and the numerical flux is not modified.

In the high-order scheme $(3.7),\left(\mathcal{S}^{t}\right)_{i, j}^{n}$ and $\left(\mathcal{S}^{f}\right)_{i, j}^{n}$ are the high-order numerical source terms of topography and friction, defined as follows:

$$
\begin{aligned}
\left(\mathcal{S}^{t}\right)_{i, j}^{n} & =\frac{1}{\left|c_{i, j}\right|} \int_{c_{i, j}}\left(\begin{array}{c}
0 \\
-g \hat{h}_{i, j}^{n} \boldsymbol{\nabla} \hat{Z}_{i, j}^{n}
\end{array}\right) d x d y \\
\left(\mathcal{S}^{f}\right)_{i, j}^{n} & =\frac{1}{\left|c_{i, j}\right|} \int_{c_{i, j}}\left(\begin{array}{c}
0 \\
-k \hat{\boldsymbol{q}}_{i, j}^{n}\left\|\hat{\boldsymbol{q}}_{i, j}^{n}\right\|\left(\hat{h}_{i, j}^{n}\right)^{-\eta}
\end{array}\right) d x d y
\end{aligned}
$$

where $\hat{Z}_{i, j}^{n}$ is the reconstruction of $Z$ within the cell $c_{i, j}$, computed from the reconstructions of $h$ and $h+Z$, and where $\hat{\boldsymbol{q}}_{i, j}^{n}=\left(\left(\hat{q}_{x}\right)_{i, j}^{n},\left(\hat{q}_{y}\right)_{i, j}^{n}\right)^{\top}$. Note that we do not compute the exact integrals involved in (3.11), but rather introduce a quadrature formula of order $(d+1)$ in the cell $c_{i, j}$. The reader is referred to [1] for more information on high-order quadrature rules on a rectangle.

\subsubsection{High-order time discretization}

Strong stability-preserving Runge-Kutta (SSPRK) methods, introduced in [44, 45], are used to increase the time accuracy of the scheme, thus providing a high-order time accuracy while retaining some robustness properties of the original scheme (3.7). The second-order $\operatorname{SSPRK}(2,2)$, third-order $\operatorname{SSPRK}(3,3)$ or fourth-order $\operatorname{SSPRK}(5,4)$ methods, described in [44,59], are used in the present study. Note that the $\operatorname{SSPRK}(2,2)$ method is nothing but Heun's method. Table 1 displays the choice of the time discretization with respect to the degree $d$.

\begin{tabular}{ccc}
\hline$d=1$ & $d=2$ & $d \geq 3$ \\
\hline $\operatorname{SSPRK}(2,2)$ & $\operatorname{SSPRK}(3,3)$ & $\operatorname{SSPRK}(5,4)$ \\
\hline
\end{tabular}

Table 1: Choice of SSPRK method with respect to the degree of the polynomial reconstruction.

For the sake of simplicity, we shall only present the SSPRK $(3,3)$ method, since the other two methods are similar. The reader is referred to [43] for an overview of the three SSPRK methods we use, as well as additional SSPRK methods. We begin by rewriting the scheme (3.7) as follows:

$$
W^{n+1}=\mathcal{H}\left(W^{n}\right)
$$

where $W^{n}$ is the vector containing the $W_{i, j}^{n}$ for $(i, j) \in \mathbb{Z}^{2}$, and $\mathcal{H}$ is a functional representing the scheme (3.7). 
With this notation, the $\operatorname{SSPRK}(3,3)$ scheme is given as follows:

$$
W^{n+1}=\frac{W^{n}+2 W^{(3)}}{3}, \quad \text { with }\left\{\begin{array}{l}
W^{(1)}=\mathcal{H}\left(W^{n}\right) \\
W^{(2)}=\mathcal{H}\left(W^{(1)}\right) \\
W^{(3)}=\mathcal{H}\left(\frac{3 W^{n}+W^{(2)}}{4}\right)
\end{array}\right.
$$

The final step in the construction of the high-order time discretization is the choice of the time step $\Delta t$. For $d \leq 3$, the time step is constrained with the classical CFL condition $(2.22)$. However, since the $\operatorname{SSPRK}(5,4)$ discretization is only fourth-order accurate in time, we have to introduce a correction of the time step for $d=4$ and $d=5$, as follows:

$$
\Delta t \leq \frac{\delta^{\frac{\max (d, 3)}{3}}}{2 \Lambda}
$$

where $\delta$ is the $2 \mathrm{D}$ mesh step, given by $\delta=\min (\Delta x, \Delta y)$. The time step condition (3.13) ensures that the time scheme will be the same order of accuracy as the space scheme.

\section{Well-balancedness recovery for the high-order scheme}

The reconstruction procedure introduced in section 3.1 causes the high-order scheme to no longer exactly preserve steady solutions, and causes non-physical oscillations when dealing with non-smooth solutions. In this section, we deal with the preservation of the steady solutions, assumed to be smooth. The oscillations are treated in the next section.

In order to recover the well-balancedness property, we propose a convex combination procedure between the first-order scheme and the high-order scheme. This convex combination recovers the well-balancedness by gradually downgrading the high-order scheme into the first-order well-balanced scheme when the solution becomes close enough to a steady state. This specific approach has been introduced in $[52,53]$ to produce a second-order well-balanced scheme (see also [49] for related work), and a proof of second-order accuracy was obtained in [11]. Here, the goal is to provide a very simple expression of the convex combination parameter that ensures high-order accuracy.

\subsection{The well-balancedness property}

We set up the convex combination of the first-order and the high-order schemes by introducing a parameter $\left(\theta_{x}\right)_{i+\frac{1}{2}, j}^{n}$ at each horizontal interface $e_{i+\frac{1}{2}, j}$ and a parameter $\left(\theta_{y}\right)_{i, j+\frac{1}{2}}^{n}$ at each vertical interface $e_{i, j+\frac{1}{2}}$. The expressions of these parameters, which will be used to define a convex combination between the first-order and the high-order schemes, shall be given later.

Recall that the first-order well-balanced scheme is given by the two steps $(2.21)-(2.24)$, while the high-order scheme is defined by (3.7). For the sake of clarity, we artificially split the high-order scheme (3.7) into two steps, the first one with the transport and the topography, and the second one with the friction, as follows:

$$
\begin{aligned}
W_{i, j}^{n+\frac{1}{2}} & =W_{i, j}^{n}-\sum_{r=1}^{N_{G}} \xi_{r} \Delta t\left[\frac{\mathcal{F}_{i+\frac{1}{2}, j, r}^{n}-\mathcal{F}_{i-\frac{1}{2}, j, r}^{n}}{\Delta x}+\frac{\mathcal{G}_{i, j+\frac{1}{2}, r}^{n}-\mathcal{G}_{i, j-\frac{1}{2}, r}^{n}}{\Delta y}\right]+\Delta t\left(\mathcal{S}^{t}\right)_{i, j}^{n}, \\
W_{i, j}^{n+1} & =W_{i, j}^{n+\frac{1}{2}}+\Delta t\left(\mathcal{S}^{f}\right)_{i, j}^{n} .
\end{aligned}
$$


We denote by $\left(\mathcal{S}^{t}\right)_{i, j}^{n}$ the second and third components of $\left(\mathcal{S}^{t}\right)_{i, j}^{n}$, defined by (3.11) and containing the topography contribution to the discharge for the high-order scheme. The first step of the high-order well-balanced scheme is merely a convex combination in each direction of the first steps of the two schemes:

$$
\begin{aligned}
W_{i, j}^{n+\frac{1}{2}}=W_{i, j}^{n} & -\frac{\Delta t}{\Delta x} \sum_{r=1}^{N_{G}} \xi_{r}\left(\left(\theta_{x}\right)_{i+\frac{1}{2}, j}^{n} \mathcal{F}_{i+\frac{1}{2}, j, r}^{n}-\left(\theta_{x}\right)_{i-\frac{1}{2}, j}^{n} \mathcal{F}_{i-\frac{1}{2}, j, r}^{n}\right) \\
& -\frac{\Delta t}{\Delta x}\left(\left(1-\left(\theta_{x}\right)_{i+\frac{1}{2}, j}^{n}\right) \mathcal{F}\left(W_{i, j}^{n}, W_{i+1, j}^{n}\right)-\left(1-\left(\theta_{x}\right)_{i-\frac{1}{2}, j}^{n}\right) \mathcal{F}\left(W_{i-1, j}^{n}, W_{i, j}^{n}\right)\right) \\
& -\frac{\Delta t}{\Delta y} \sum_{r=1}^{N_{G}} \xi_{r}\left(\left(\theta_{y}\right)_{i, j+\frac{1}{2}}^{n} \mathcal{G}_{i, j+\frac{1}{2}, r}^{n}-\left(\theta_{y}\right)_{i, j-\frac{1}{2}}^{n} \mathcal{G}_{i, j-\frac{1}{2}, r}^{n}\right) \\
& -\frac{\Delta t}{\Delta y}\left(\left(1-\left(\theta_{y}\right)_{i, j+\frac{1}{2}}^{n}\right) \mathcal{G}\left(W_{i, j}^{n}, W_{i, j+1}^{n}\right)-\left(1-\left(\theta_{y}\right)_{i, j-\frac{1}{2}}^{n}\right) \mathcal{G}\left(W_{i, j-1}^{n}, W_{i, j}^{n}\right)\right) \\
& +\Delta t\left(\boldsymbol{\theta}_{i, j}^{n} \cdot\left(\mathcal{S}^{t}\right)_{i, j}^{n}+\left(1-\boldsymbol{\theta}_{i, j}^{n}\right) \cdot\left(\boldsymbol{S}^{t}\right)_{i, j}^{n}\right)
\end{aligned}
$$

where $\boldsymbol{\theta}_{i, j}^{n}:=\left(\left(\theta_{x}\right)_{i, j}^{n},\left(\theta_{y}\right)_{i, j}^{n}\right)^{\top}$ is a convex parameter on the cell $c_{i, j}$, whose expression with respect to $\left(\theta_{x}\right)_{i \pm \frac{1}{2}, j}^{n}$ and $\left(\theta_{y}\right)_{i, j \pm \frac{1}{2}}^{n}$ will also be given later.

Concerning the updated water heights, we take $h_{i, j}^{n+1}=h_{i, j}^{n+\frac{1}{2}}$, since the last step is devoted to the friction source term and therefore has no impact on the water height. We denote by $\left(\mathcal{S}^{f}\right)_{i, j}^{n}$ the second and third components of $\left(\mathcal{S}^{f}\right)_{i, j}^{n}$, as defined by (3.11). Following (2.24), let $\left(\boldsymbol{q}_{\mathrm{WB}}\right)_{i, j}^{n+1}$ be the vector containing the discharge obtained after the second step of the first-order scheme. The second step of the high-order well-balanced scheme consists in the convex combination of the two second steps, as follows:

$$
\boldsymbol{q}_{i, j}^{n+1}=\boldsymbol{\theta}_{i, j}^{n} \cdot\left(\boldsymbol{q}_{i, j}^{n+\frac{1}{2}}+\Delta t\left(\mathcal{S}^{f}\right)_{i, j}^{n}\right)+\left(1-\boldsymbol{\theta}_{i, j}^{n}\right) \cdot\left(\boldsymbol{q}_{\mathrm{WB}}\right)_{i, j}^{n+1} .
$$

In the two-step scheme $(4.1)-(4.2)$, if $\left(\theta_{x}\right)_{i \pm \frac{1}{2}, j}^{n}$ and $\left(\theta_{y}\right)_{i, j \pm \frac{1}{2}}^{n}$ are close to 1 , then the high-order scheme is preferred, while the first-order well-balanced scheme is used if $\left(\theta_{x}\right)_{i \pm \frac{1}{2}, j}^{n}$ and $\left(\theta_{y}\right)_{i, j \pm \frac{1}{2}}^{n}$ are close to 0 .

In order to recover the high-order accuracy in time, we apply the relevant SSPRK procedure with respect to the degree of the polynomial reconstruction, according to table 1. For instance, if $d=2$, the $\operatorname{SSPRK}(3,3)$ method (3.12) is used.

\subsection{A high-order accurate convex combination}

The convex combination detailed in section 4.1 is performed in each cell $c_{i, j}$, while computing the numerical fluxes and the numerical source terms. The only remaining unknowns are the parameters $\left(\theta_{x}\right)_{i+\frac{1}{2}, j}^{n}$ and $\left(\theta_{y}\right)_{i, j+\frac{1}{2}}^{n}$. Note that, if $\left(\theta_{x}\right)_{i \pm \frac{1}{2}, j}^{n}$ and $\left(\theta_{y}\right)_{i, j \pm \frac{1}{2}}^{n}$ are approximations of 1 up to order $\Delta x^{d+1}$ in each direction, then the two-step scheme with convex combination (4.1) - (4.2) is automatically of order $(d+1)$, like the fully high-order scheme (3.7).

In the remainder of this section, we derive an expression of the convex combination parameter in the $x$ direction $\left(\theta_{x}\right)_{i+\frac{1}{2}, j}^{n}$. The expressions in the $y$-direction are obtained in a similar fashion. Let us define a steady 
state detector $\varepsilon_{x}$ in the $x$-direction between two states $W_{L}$ and $W_{R}$, as follows:

$$
\begin{aligned}
\varepsilon_{x}\left(W_{L}, W_{R}, Z_{L}, Z_{R}, x_{L}, x_{R}\right) & =\sqrt{\left(\psi_{x}^{t}\left(W_{R}, Z_{R}\right)-\psi_{x}^{t}\left(W_{L}, Z_{L}\right)\right)^{2}+\left(\left(q_{x}\right)_{R}-\left(q_{x}\right)_{L}\right)^{2}+\frac{1}{2}\left(\left(q_{y}\right)_{R}^{2}+\left(q_{y}\right)_{L}^{2}\right)} \\
& \times \sqrt{\left(\psi_{x}^{f}\left(W_{R}, x_{R}\right)-\psi_{x}^{f}\left(W_{L}, x_{L}\right)\right)^{2}+\left(\left(q_{x}\right)_{R}-\left(q_{x}\right)_{L}\right)^{2}+\frac{1}{2}\left(\left(q_{y}\right)_{R}^{2}+\left(q_{y}\right)_{L}^{2}\right)}
\end{aligned}
$$

where $\psi_{x}^{t}(W, Z)$ and $\psi_{x}^{f}(W, x)$ are respectively defined by the algebraic expressions of the topography and friction steady states (1.11) and (1.13), as follows:

$$
\begin{aligned}
& \psi_{x}^{t}(W, Z)=\frac{q^{2}}{2 h^{2}}+g(h+Z) \\
& \psi_{x}^{f}(W, x)=-q^{2} \frac{h^{\eta-1}}{\eta-1}+g \frac{h^{\eta+2}}{\eta+2}+k q|q| x
\end{aligned}
$$

Note that this definition ensures that the steady state detector $\varepsilon_{x}$ vanishes as soon as a steady state with either topography or friction is detected between the states $W_{L}$ and $W_{R}$ in the $x$-direction, that is to say as soon as the pairs $\left(h_{L},\left(q_{x}\right)_{L}, Z_{L}\right)$ and $\left(h_{R},\left(q_{x}\right)_{R}, Z_{R}\right)$ define a 1 D steady state, and that $\left(q_{y}\right)_{L}=\left(q_{y}\right)_{R}=0$.

We define the convex combination parameter $\left(\theta_{x}\right)_{i+\frac{1}{2}, j}^{n}$ at the interface $e_{i+\frac{1}{2}, j}$ by:

$$
\left(\theta_{x}\right)_{i+\frac{1}{2}, j}^{n}=\frac{\left(\varepsilon_{x}\right)_{i+\frac{1}{2}, j}^{n}}{\left(\varepsilon_{x}\right)_{i+\frac{1}{2}, j}^{n}+\left(\frac{\Delta x}{\mathcal{L}_{x}}\right)^{k}}
$$

with $\left(\varepsilon_{x}\right)_{i+\frac{1}{2}, j}^{n}=\varepsilon_{x}\left(W_{i, j}^{n}, W_{i+1, j}^{n}, Z_{i, j}, Z_{i+1, j}, x_{i, j}, x_{i+1, j}\right), \mathcal{L}_{x}$ a characteristic length and $k \geq d+1$. The convex combination parameter in the $x$-direction $\left(\theta_{x}\right)_{i, j}^{n}$ within the cell $c_{i, j}$ is then defined as follows:

$$
\left(\theta_{x}\right)_{i, j}^{n}=\sqrt{\frac{1}{2}\left(\left(\left(\theta_{x}\right)_{i-\frac{1}{2}, j}^{n}\right)^{2}+\left(\left(\theta_{x}\right)_{i+\frac{1}{2}, j}^{n}\right)^{2}\right)} .
$$

Proposition 4. The convex combination parameter in the $x$-direction $\left(\theta_{x}\right)_{i, j}^{n}$, given by (4.6), satisfies the following properties:

- $\left(\theta_{x}\right)_{i, j}^{n}$ vanishes as soon as $W_{i-1, j}^{n}, W_{i, j}^{n}$ and $W_{i+1, j}^{n}$ define a steady state;

- If $W_{i-1, j}^{n}, W_{i, j}^{n}$ and $W_{i+1, j}^{n}$ do not define a steady state, then $\left(\theta_{x}\right)_{i, j}^{n}$ is an approximation of 1 up to $\Delta x^{d+1}$.

Proof. Equipped with the definition (4.3) of the steady state detector $\varepsilon_{x}$, we immediately obtain that $\left(\varepsilon_{x}\right)_{i+\frac{1}{2}, j}^{n}$ vanishes as soon as $W_{i, j}^{n}$ and $W_{i+1, j}^{n}$ define a steady state. Therefore, in this case, $\left(\theta_{x}\right)_{i+\frac{1}{2}, j}^{n}$ given by $(4.5)$, also vanishes, which ensures that $\left(\theta_{x}\right)_{i, j}^{n}$ vanishes as soon as $W_{i-1, j}^{n}, W_{i, j}^{n}$ and $W_{i+1, j}^{n}$ define a steady state, and proves the first property.

The second property is proven by arguing a Taylor expansion of the expression (4.5). Indeed, let us assume that $W_{i-1, j}^{n}, W_{i, j}^{n}$ and $W_{i+1, j}^{n}$ do not define a steady state, i.e. that $\left(\varepsilon_{x}\right)_{i \pm \frac{1}{2}, j}^{n} \neq 0$. Then, $\left(\theta_{x}\right)_{i \pm \frac{1}{2}, j}^{n}$ can be rewritten as follows:

$$
\left(\theta_{x}\right)_{i \pm \frac{1}{2}, j}^{n}=\frac{1}{1+\mathcal{C}_{i, j}^{n} \Delta x^{k}}, \quad \text { with } \quad \mathcal{C}_{i, j}^{n}=\frac{1}{\left(\varepsilon_{x}\right)_{i \pm \frac{1}{2}, j}^{n} \mathcal{L}_{x}^{k}}
$$


Since $\mathcal{C}_{i, j}^{n}$ is a constant independent of $\Delta x$, we get

$$
\left(\theta_{x}\right)_{i \pm \frac{1}{2}, j}^{n}=1-\mathcal{C}_{i, j}^{n} \Delta x^{k}+\mathcal{O}\left(\Delta x^{2 k}\right)=1+\mathcal{O}\left(\Delta x^{d+1}\right),
$$

since $k$ is such that $k \geq d+1$. Then, arguing another Taylor expansion in the definition (4.6) of $\left(\theta_{x}\right)_{i, j}^{n}$, we immediately get $\left(\theta_{x}\right)_{i, j}^{n}=1+\mathcal{O}\left(\Delta x^{d+1}\right)$.

In the $y$-direction, the convex combination parameter $\left(\theta_{y}\right)_{i, j}^{n}$ is obtained in a similar fashion. Equipped with the expressions of $\left(\theta_{x}\right)_{i+\frac{1}{2}, j}^{n}$ and $\left(\theta_{y}\right)_{i, j+\frac{1}{2}}^{n}$, the high-order well-balanced scheme (4.1) - (4.2) is complete, and its properties are summarized in the following result.

Theorem 5. The scheme (4.1) - (4.2), equipped with the convex combination parameter defined in section 4.2, is well-balanced in the $x$-and $y$-directions and high-order accurate.

Proof. Since the convex combination parameter vanishes as soon as a steady state in the $x$ - and $y$-directions is detected, the convex combination scheme (4.1) - (4.2) reverts to the first-order scheme in this case, which is well-balanced according to theorem 3. In addition, since the convex combination parameter is nothing but an approximation of 1 up to $\Delta x^{d+1}$ according to proposition 4 , the resulting convex combination scheme is necessarily high-order accurate far from a steady state. The proof is thus concluded.

\section{The MOOD method}

The previous section proposes an adaptation of the high-order scheme to recover the well-balancedness. However, this high-order accuracy comes with the loss of the non-negativity property, and the numerical solutions obtained with this scheme may present spurious oscillations around discontinuities (see [64, 51] for instance).

To address this issue, we use MOOD techniques (see [28, 35, 36] for an overview of this method, and $[9,38$, 30, 29] for more recent applications, related to the shallow water equations and dry/wet transitions). Classical MOOD limiters, detailed below, are applied to preserve the non-negativity as well as to prevent the scheme from creating non-physical oscillations.

\subsection{Overview of the MOOD method}

The goal of the MOOD procedure is to recover the essential stability properties of a first-order scheme, for instance its robustness, by detecting whether the properties are verified by the high-order approximation. If this verification fails in some cell, the degree of the approximation is lowered in this cell, until the properties are satisfied. In this work, we use a more direct version of this method, by switching to the first-order scheme as soon as the verification fails, instead of progressively downgrading the polynomial degree. This choice is motivated by the fact that only the first-order scheme is well-balanced, and the well-balancedness preservation for the high-order scheme requires directly switching to the first-order scheme, as explained in section 4 .

The core of the MOOD method is in the choice of the properties that need to be satisfied by the high-order scheme, and in their detection. Detection criteria are commonly used within the MOOD procedure. Note that the detection criteria in section 5.1 have already been introduced in [38, 29] (and references therein). In this section, we recall these criteria for the sake of completeness.

We introduce the notation $W^{\star}$ for the candidate solution, i.e. the solution obtained from $W^{n}$ using the highorder well-balanced scheme presented in section 4. This candidate solution is then tested against the following criteria, to determine the cells where it is not acceptable. 


\subsubsection{Physical Admissibility Detector (PAD)}

The PAD determines whether the approximate solution is out of the admissible states space $\Omega$. In the case of the shallow water equations, we check whether the water height is non-negative, and state that the PAD criterion fails within the cell $c_{i, j}$ if

$$
h_{i, j}^{\star}<0 .
$$

Let us underline that, equipped with the PAD, the high-order scheme is non-negativity preserving.

\subsubsection{Discrete Maximum Principle (DMP)}

Although the PAD ensures the non-negativity preservation, it does not prevent spurious oscillations from appearing in the vicinity of discontinuities. To address this issue, we use the DMP criterion to check for potential oscillations. Let $\nu_{i, j}$ be the set of cells connected to $c_{i, j}$ with an edge or a vertex. The DMP criterion fails if one of the following three checks is not fulfilled:

$$
\begin{aligned}
\min _{l \in \nu_{i, j}}\left(h_{l}+Z_{l}\right)-\varepsilon_{h} \leq h_{i, j}^{\star}+Z_{i, j}^{\star} \leq \min _{l \in \nu_{i, j}}\left(h_{l}+Z_{l}\right)+\varepsilon_{h}, \\
\min _{l \in \nu_{i, j}}\left(\left(q_{x}\right)_{l}\right)-\varepsilon_{q} \leq\left(q_{x}\right)_{i, j}^{\star} \leq \min _{l \in \nu_{i, j}}\left(\left(q_{x}\right)_{l}\right)+\varepsilon_{q}, \\
\min _{l \in \nu_{i, j}}\left(\left(q_{y}\right)_{l}\right)-\varepsilon_{q} \leq\left(q_{y}\right)_{i, j}^{\star} \leq \min _{l \in \nu_{i, j}}\left(\left(q_{y}\right)_{l}\right)+\varepsilon_{q},
\end{aligned}
$$

where $\varepsilon_{h}$ and $\varepsilon_{q}$ are used to reduce the risk of oscillation overdetection, mainly due to floating-point truncation errors. In practice, we usually take $\varepsilon_{h}=\varepsilon_{q}=\delta^{3}$, with $\delta=\min (\Delta x, \Delta y)$.

\subsubsection{Detecting physical oscillations: the u2 criterion}

The DMP criterion (5.1) can detect and eliminate physical extrema, thus resulting in a false positive that reduces the accuracy of the scheme. Therefore, we add another criterion to detect whether an extremum is physically admissible, namely the $u 2$ criterion. It uses the second derivative of the polynomial reconstruction $\hat{\varphi}_{i, j}(x, y ; 2)$. Note that, since $\hat{\varphi}_{i, j}(x, y ; 2)$ is a second-degree polynomial, its second derivative is constant. In practice, we take $\varphi \in\left\{h+Z, q_{x}, q_{y}\right\}$. We then define the following curvatures on the cell $c_{i, j}$ :

$$
\begin{array}{ll}
\mathcal{X}_{i, j}^{\min }=\min _{l \in \nu_{i, j}}\left(\partial_{x x} \hat{\varphi}_{i, j}, \partial_{x x} \hat{\varphi}_{l}\right), & \mathcal{X}_{i, j}^{\max }=\max _{l \in \nu_{i, j}}\left(\partial_{x x} \hat{\varphi}_{i, j}, \partial_{x x} \hat{\varphi}_{l}\right), \\
\mathcal{Y}_{i, j}^{\min }=\min _{l \in \nu_{i, j}}\left(\partial_{y y} \hat{\varphi}_{i, j}, \partial_{y y} \hat{\varphi}_{l}\right), & \mathcal{Y}_{i, j}^{\max }=\max _{l \in \nu_{i, j}}\left(\partial_{y y} \hat{\varphi}_{i, j}, \partial_{y y} \hat{\varphi}_{l}\right) .
\end{array}
$$

Equipped with the curvatures, we define three criteria, which are combined to form the $u 2$ criterion (see $[38,29])$. First, the plateau detector focuses on the micro-oscillations, and is defined as follows:

$$
\max \left(\left|\mathcal{X}_{i, j}^{\min }\right|,\left|\mathcal{X}_{i, j}^{\max }\right|,\left|\mathcal{Y}_{i, j}^{\min }\right|,\left|\mathcal{Y}_{i, j}^{\max }\right|\right) \leq \delta
$$

Next, the local oscillation detector is given by:

$$
\mathcal{X}_{i, j}^{\min } \mathcal{X}_{i, j}^{\max } \geq-\delta \quad \text { and } \quad \mathcal{Y}_{i, j}^{\min } \mathcal{Y}_{i, j}^{\max } \geq-\delta
$$

The third criterion involves a smoothness detector, given as follows, to assess whether the solution is locally smooth:

$$
\frac{1}{2} \leq \frac{\min \left(\left|\mathcal{X}_{i, j}^{\min }\right|,\left|\mathcal{X}_{i, j}^{\max }\right|\right)}{\max \left(\left|\mathcal{X}_{i, j}^{\min }\right|,\left|\mathcal{X}_{i, j}^{\max }\right|\right)} \leq 1 \quad \text { and } \quad \frac{1}{2} \leq \frac{\min \left(\left|\mathcal{Y}_{i, j}^{\min }\right|,\left|\mathcal{Y}_{i, j}^{\max }\right|\right)}{\max \left(\left|\mathcal{Y}_{i, j}^{\min }\right|,\left|\mathcal{Y}_{i, j}^{\max }\right|\right)} \leq 1 .
$$


The $u 2$ criterion is finally defined as a combination of these three detectors. Indeed, if a plateau is detected by (5.2) or if the solution is considered locally smooth by (5.4), then the DMP criterion becomes irrelevant and the $u 2$ criterion succeeds. On the contrary, if a local oscillation is detected by (5.3), then the $u 2$ criterion fails.

\subsubsection{The detector chain}

Equipped with these detectors, we state the order in which the detectors are checked. To address this issue, we introduce the Cell Polynomial Degree (CPD) as an integer, associated to a cell $c_{i, j}$, such that $\operatorname{CPD}(i, j) \in\{0, d\}$. If $\operatorname{CPD}(i, j)=0$, then the first-order scheme is used in the cell $c_{i, j}$. On the contrary, if $\operatorname{CPD}(i, j)=d$, then the high-order scheme is used within cell $c_{i, j}$. Figure 4 displays the detector chain, and the effect of each detector on the CPD.

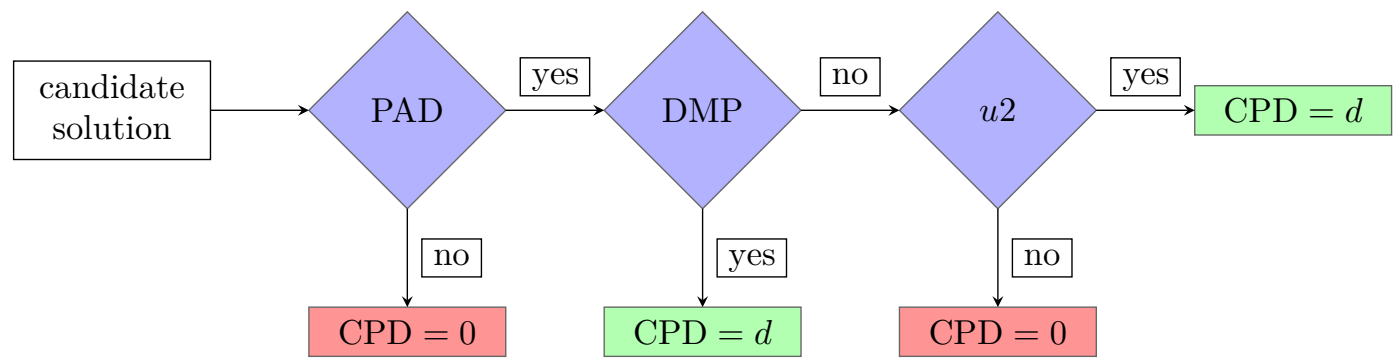

Figure 4: The MOOD detector chain.

At the end of the chain, if $\operatorname{CPD}(i, j)=d$, the candidate solution is declared eligible in the cell $c_{i, j}$, and is accepted as the updated approximate solution $W^{n+1}$. If one of the criteria did fail, then $\operatorname{CPD}(i, j)=0$ is set to 0 and the candidate solution is discarded in the cell $c_{i, j}$ and its neighbors. If that is the case, a new candidate solution is computed using a polynomial reconstruction whose degree in cell $c_{i, j}$ is equal to $\operatorname{CPD}(i, j)$. Note that, if a cell and its neighbors are declared eligible, there is no need to compute a new candidate solution in these cells.

\subsection{Algorithm for the high-order well-balanced scheme with MOOD detection}

According to section 4, the stationarity of a numerical solution is determined at time $t^{n}$, before any update computations. Hence, when a steady solution is detected, the first-order scheme is used and the MOOD limiters described in section 5.1 become unnecessary. Therefore, we first check the stationarity of the numerical solution, before applying the MOOD procedure. Then, the admissibility of the reconstruction is checked twice, once when computing the reconstructed heights at the Gauss points, and once when computing the numerical approximation of the mean of the friction source term. The full MOOD procedure, applied to the high-order well-balanced scheme (4.1) - (4.2), is detailed below. Note that, to prevent non-physical solutions, this MOOD procedure is applied at each step of the SSPRK time discretization.

Algorithm 6. For a single iteration in time of the SSPRK time discretization, the MOOD loop reads as follows.

1. For each interface $e_{i+\frac{1}{2}, j}$ (resp. $\left.e_{i, j+\frac{1}{2}}\right)$, compute the correction parameters $\left(\theta_{x}\right)_{i+\frac{1}{2}, j}^{n}$ or $\left(\theta_{y}\right)_{i, j+\frac{1}{2}}^{n}$ according to (4.5). Then, for each cell $c_{i, j}$, compute $\boldsymbol{\theta}_{i, j}^{n}$ with (4.6).

2. For each cell $c_{i, j}$, initialize $C P D(i, j)=d$. If $\boldsymbol{\theta}_{i, j}^{n}=\mathbf{0}$, then $C P D(i, j)=0$. 
3. For each cell $c_{i, j}$, if $C P D(i, j)>0$, compute the interface reconstruction. If $\hat{h}_{i, j}^{n}(\zeta)<0$ for some edge Gauss point $\zeta$, then the reconstruction is rejected in that cell, and we set $C P D(i, j)=0$.

4. For each cell $c_{i, j}$, if $C P D(i, j)>0$, compute the cell reconstruction. If $\hat{h}_{i, j}^{n}(\zeta)<0$ for some cell Gauss point $\zeta$, then the reconstruction is rejected in that cell, and we set $C P D(i, j)=0$.

5. Equipped with the new CPD map, compute the candidate solution $W^{\star}$, using the high-order well-balanced scheme (4.1) - (4.2).

6. Apply the detection process displayed in figure 4 to compute a potentially new CPD map and to decide whether to accept the candidate solution. If the candidate solution is rejected, go to step 5 with the new CPD map. Otherwise, go to step $\%$

7. The candidate solution is accepted, and we set $W^{n+1}=W^{\star}$.

\section{Numerical experiments}

This last section is devoted to numerical experiments, designed to highlight the essential properties of the scheme. The following notation is introduced to concisely label the schemes to be tested.

- The scheme that uses a polynomial reconstruction of degree $d$, i.e. whose order of accuracy is $(d+1)$, is denoted by $\mathbb{P}_{d}$, including the first-order well-balanced scheme. For $d \geq 1$, the $\mathbb{P}_{d}$ scheme includes the MOOD detection loop from section 5.1, but not the well-balancedness correction from section 5.2.

- For $d \geq 1$, the $\mathbb{P}_{d}$ scheme equipped with the well-balancedness correction from section 5.2 is denoted by $\mathbb{P}_{d}^{W B}$.

In addition, in order to assess the well-balancedness and the high-order accuracy of the scheme, we shall evaluate the error between the exact solution $W^{e x}(t, x, y)$ and the approximate solution. Consider a uniform Cartesian mesh made of $N=N_{x} \times N_{y}$ cells. We denote by $W_{i, j}^{e x}$ the average of the exact solution over the cell $c_{i, j}$ at time $t$, as follows:

$$
W_{i, j}^{e x}(t)=\frac{1}{\Delta x \Delta y} \int_{c_{i, j}} W^{e x}(t, x, y) d x d y .
$$

Equipped with this notation, we compute the errors in $L^{1}, L^{2}$ and $L^{\infty}$ norms between with $W_{i, j}^{n}$, the approximate solution at time $t^{n}$, and the exact solution $W_{i, j}^{e x}\left(t^{n}\right)$ :

$$
\begin{aligned}
& L^{1} \text { error: } \frac{1}{N} \sum_{i=1}^{N_{x}} \sum_{j=1}^{N_{y}}\left|W_{i, j}^{n}-W_{i, j}^{e x}\left(t^{n}\right)\right|, \\
& L^{2} \text { error: } \sqrt{\frac{1}{N} \sum_{i=1}^{N_{x}} \sum_{j=1}^{N_{y}}\left(W_{i, j}^{n}-W_{i, j}^{e x}\left(t^{n}\right)\right)^{2}}, \\
& L^{\infty} \text { error: } \max _{\substack{1 \leq i \leq N_{x} \\
1 \leq j \leq N_{y}}}\left|W_{i, j}^{n}-W_{i, j}^{e x}\left(t^{n}\right)\right| .
\end{aligned}
$$

The evaluation of $W_{i, j}^{e x}(t)$ for all cells $c_{i, j}$ is achieved by using a quadrature rule of the same order as the scheme (see [1] for instance). To assess the well-balancedness and the accuracy of the scheme, we evaluate these errors at the final physical time $t_{\text {end }}$. 
Let us recall here that, given $\Delta x$ and $\Delta y$, the time step $\Delta t$ is constrained by the CFL-like condition (3.13). In practice, we take the following time step:

$$
\Delta t=\frac{\delta^{\frac{\max \left(\mathrm{CPD}_{\max }, 3\right)}{3}}}{2 \Lambda}
$$

where $\mathrm{CPD}_{\max }:=\max _{i, j}[\mathrm{CPD}(i, j)]$, where $\delta=\min (\Delta x, \Delta y)$ and where $\Lambda$ is the maximum of all characteristic velocities at each interface. As soon as the solution is steady on the whole mesh, we get $\mathrm{CPD}_{\max }=0$ and the first-order scheme is used everywhere. In this case, the time step (6.1) degenerates towards the time step of the first-order scheme (2.22). This saves a considerable amount of CPU time without modifying the accuracy of the scheme, which is exact up to machine precision on steady solutions.

Finally, unless otherwise specified, the two parameters from the well-balancedness detection are defined as follows: the characteristic length $\mathcal{L}_{x}$ is taken as the length of the domain, and we take $k=d+1$. In addition, we set $g=9.81 \mathrm{~m} \mathrm{~s}^{-2}$ and we recall that $\eta=7 / 3$. To deal with vanishing water heights, the velocity $\boldsymbol{u}$ is defined according to the machine precision $\varepsilon_{m}$, where $\varepsilon_{m} \simeq 2.22 \times 10^{-16}$ for double-precision floating-point numbers. Namely, if $h<\varepsilon_{m}$, we set $\boldsymbol{u}=\mathbf{0}$; otherwise, we set $\boldsymbol{u}=\boldsymbol{q} / h$.

We first propose in section 6.1 several numerical experiments designed to assess the well-balancedness of the $\mathbb{P}_{d}^{W B}$ scheme, namely the preservation of the $2 \mathrm{D}$ lake at rest and of $1 \mathrm{D}$ moving steady states. Then, section 6.2 is dedicated to the high-order accuracy on 2D exact solutions. Dam-break experiments are tackled in section 6.3, while the simulation of the 2011 Tōhoku tsunami is carried out in section 6.4.

\subsection{Well-balancedness assessment}

We perform numerical experiments to assess the well-balancedness of the scheme. The first experiment concerns the preservation of a lake at rest steady state with a dry area, and the second one focuses on capturing a one-dimensional moving steady state with friction and topography that has been perturbed.

\subsubsection{Preservation of the lake at rest}

We begin the well-balancedness numerical experiments with the preservation of a lake at rest steady state. This experiment involves a nonzero Manning coefficient $k=10$, nonconstant topography and a dry area. On the space domain $[0,1] \times[0,1]$, the topography is given by:

$$
Z(x, y)=\sqrt{x^{2}+y^{2}} .
$$

The water height and the discharge are chosen according to (1.7), ensuring that $h$ stays nonnegative, as follows:

$$
h(t, x, y)=(1-Z(x, y))_{+} \quad \text { and } \quad \boldsymbol{q}(t, x, y)=\mathbf{0} .
$$

Note that this steady solution contains transitions between dry and wet areas. The exact solution is prescribed as both initial and boundary conditions.

In order to highlight the relevance of the well-balancedness correction, the simulation is carried out using the first-order scheme and the sixth-order scheme, with and without correction. The results of the experiment are reported in table 2 , for $2500(50 \times 50)$ cells and at time $t_{\text {end }}=0.1 \mathrm{~s}$. For this simulation, we set the cut-off constant $C$ defined in (2.10) to $C=+\infty$. Numerically, we take $C$ as the upper bound of the double-precision floating-point numbers. 


\begin{tabular}{|c|c|c|c|c|c|c|c|}
\hline \multirow[b]{2}{*}{ scheme } & \multicolumn{3}{|c|}{$h+Z$} & \multicolumn{3}{|c|}{$\|\boldsymbol{q}\|$} & \multirow[b]{2}{*}{ CPU time $(\mathrm{s})$} \\
\hline & $L^{1}$ & $L^{2}$ & $L^{\infty}$ & $L^{1}$ & $L^{2}$ & $L^{\infty}$ & \\
\hline $\mathbb{P}_{0}$ & $5.76 \mathrm{e}-18$ & $2.53 \mathrm{e}-17$ & $2.22 \mathrm{e}-16$ & $5.68 \mathrm{e}-17$ & $1.05 \mathrm{e}-16$ & $7.44 \mathrm{e}-16$ & 0.04259 \\
\hline $\mathbb{P}_{5}$ & $5.85 \mathrm{e}-05$ & $1.60 \mathrm{e}-04$ & $1.51 \mathrm{e}-03$ & $7.98 \mathrm{e}-05$ & $2.62 \mathrm{e}-04$ & $7.54 \mathrm{e}-03$ & 4.205 \\
\hline $\mathbb{P}_{5}^{\mathrm{WB}}$ & $2.71 \mathrm{e}-17$ & $6.95 \mathrm{e}-17$ & $6.66 \mathrm{e}-16$ & $2.59 \mathrm{e}-16$ & $3.77 \mathrm{e}-16$ & $2.13 \mathrm{e}-15$ & 0.3012 \\
\hline
\end{tabular}

Table 2: Free surface error, discharge norm error and CPU time for the lake at rest experiment, using each of the three schemes.

From table 2, we observe that the first-order scheme indeed exactly preserves the lake at rest. However, the sixth-order $\mathbb{P}_{5}$ scheme, as expected, does not exactly preserve the lake at rest but instead gives a sixth-order approximation of this steady state. The relevance of the correction is thus highlighted here, since it allows to recover the exact lake at rest steady state. In addition, the correction reduces the CPU time taken by the $\mathbb{P}_{5}^{\mathrm{WB}}$ scheme by a factor of about 14 compared to the $\mathbb{P}_{5}$ scheme. This is due to the fact that, since the solution is steady, the detection procedure forces the use of the first-order scheme. The difference in CPU time between the $\mathbb{P}_{5}^{\mathrm{WB}}$ and $\mathbb{P}_{0}$ schemes comes from the definition (6.1) of the time step, as well as from the SSPRK3 time integrator (3.12).

\subsubsection{Subcritical steady flow over a bump without friction}

To assess the well-balancedness in the $x$ - and $y$-directions, we consider the well-known Goutal and Maurel test case from [46] We perform this experiment in the $x$-direction; of course, the same conclusion is reached by considering the test case in the $y$-direction.

The initial conditions for this experiment consist in a lake at rest over a topography with a bump, given on the domain $(0,25) \times(0,1)$ by:

$$
Z(x, y)=\max \left(\left[0.2-0.05(x-10)^{2}\right], 0\right) .
$$

The initial free surface is given by $h(0, x, y)+Z(x, y)=2$, and the initial discharge is set to zero. The friction is canceled, and we take $k=0$.

The main feature of this experiment is that the final steady state is obtained following a transient state, which is governed by the boundary conditions. Neumann boundary conditions are prescribed on each boundary and each variable, except on the left boundary where the $x$-discharge is such that $q_{x}(t, 0, y)=4.42$, and on the right boundary where the height is set to $h(t, 25, y)=2$. These boundary conditions enable the eventual formation of a subcritical moving steady state. Therefore, this test case not only checks whether the well-balanced scheme under consideration is able to preserve a given steady state, but also if it is possible to capture a steady state obtained after a transient state. In [52], the first-order well-balanced scheme was shown to capture this subcritical steady state, and gives evidence that the well-balancedness correction of the $\mathbb{P}_{5}^{\mathrm{WB}}$ scheme should also capture the steady solution.

The simulation is carried out on $100=100 \times 1$ cells, using the first-order scheme and the sixth-order scheme, with and without correction. Regarding the correction, we take $\mathcal{L}_{x}=0.5$. The errors are presented in table 3 at the final time $t_{\text {end }}=500 \mathrm{~s}$, where $\psi_{x}^{t}$ is the topography steady state detector in the $x$-direction given by (4.4a), which becomes constant once a steady state is reached. We correctly recover the expected behavior, that is to say both the $\mathbb{P}_{0}$ and $\mathbb{P}_{5}^{\mathrm{WB}}$ schemes capture the subcritical steady state up to the machine precision, while the $\mathbb{P}_{5}$ scheme merely approximates this steady state. The trend in CPU time reported in the previous experiment is still observed, with the $\mathbb{P}_{5}^{\mathrm{WB}}$ scheme switching to the $\mathbb{P}_{0}$ scheme once the steady state is reached. 


\begin{tabular}{|c|c|c|c|c|c|c|c|}
\hline \multirow[b]{2}{*}{ scheme } & \multicolumn{3}{|c|}{$\psi_{x}^{t}$} & \multicolumn{3}{|c|}{$\|\boldsymbol{q}\|$} & \multirow[b]{2}{*}{ CPU time $(\mathrm{s})$} \\
\hline & $L^{1}$ & $L^{2}$ & $L^{\infty}$ & $L^{1}$ & $L^{2}$ & $L^{\infty}$ & \\
\hline $\mathbb{P}_{0}$ & $1.03 \mathrm{e}-13$ & $1.85 \mathrm{e}-13$ & $1.41 \mathrm{e}-13$ & $6.69 \mathrm{e}-14$ & $7.74 \mathrm{e}-14$ & $1.41 \mathrm{e}-13$ & 0.7412 \\
\hline $\mathbb{P}_{5}$ & $2.14 \mathrm{e}-04$ & $1.03 \mathrm{e}-03$ & $9.92 \mathrm{e}-03$ & $2.12 \mathrm{e}-04$ & $5.44 \mathrm{e}-04$ & $2.11 \mathrm{e}-03$ & 42.43 \\
\hline $\mathbb{P}_{5}^{\mathrm{WB}}$ & $1.05 \mathrm{e}-12$ & $1.88 \mathrm{e}-12$ & $4.34 \mathrm{e}-12$ & $6.75 \mathrm{e}-13$ & $8.25 \mathrm{e}-13$ & $1.53 \mathrm{e}-12$ & 16.42 \\
\hline
\end{tabular}

Table 3: Error on $\psi_{x}^{t}$, error on $\|\boldsymbol{q}\|$ and CPU time for the subcritical Goutal and Maurel test case, using each of the three schemes. Note that a steady solution is exactly captured after a transient state.

\subsubsection{Perturbed steady state with friction over a flat topography}

We now consider a numerical experiment designed to check the well-balancedness with respect to the friction source term. To that end, we take $k=1$ and a flat topography $Z(x, y)=0$. The initial water height derives from solving the equation $\psi_{x}^{f}=0.02$, with $q_{x}(x, y)=-0.5$ and $q_{y}(x, y)=0$, where $\psi_{x}^{f}$, defined by $(4.4 \mathrm{~b})$, is constant for a friction steady state. This initial condition $W^{\text {steady }}(x, y)$, therefore, represents a steady state at rest in the $x$-direction for the friction source term, and it should be preserved by the well-balanced schemes. Note that the same conclusion is obtained for the $y$-direction.

To study the capture, rather than the preservation, of a friction steady state we add a perturbation to the steady solution $W^{\text {steady }}(x, y)$. The height is perturbed as follows:

$$
\begin{cases}h(0, x, y)=h^{\text {steady }}(x, y)+0.05 & \text { if } x \in\left(\frac{3}{7}, \frac{4}{7}\right), \\ h(0, x, y)=h^{\text {steady }}(x, y) & \text { otherwise }\end{cases}
$$

while the following perturbation is applied to the $x$-discharge:

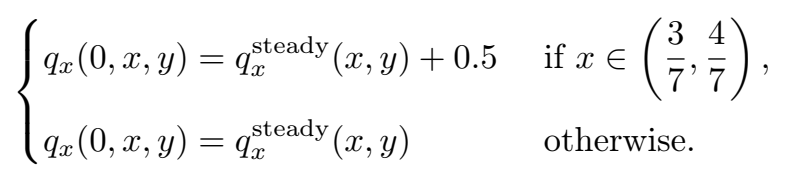

We prescribe the exact steady solution as inhomogeneous Dirichlet boundary conditions for this experiment. This ensures that the correct steady state is recovered once the perturbation is dissipated, i.e. once the perturbation has lest the space domain. Thus, the test case enables to test the capture of the resulting friction-only steady state, obtained after a transient state. A similar experiment was performed in [53], where the first-order scheme was shown to capture this steady state at machine precision.

We carry out the simulation on $100=100 \times 1$ cells with the $\mathbb{P}_{0}, \mathbb{P}_{5}$ and $\mathbb{P}_{5}^{\mathrm{WB}}$ schemes, until the final time $t_{\text {end }}=5 \mathrm{~s}$. For the $\mathbb{P}_{5}^{\mathrm{WB}}$ scheme, we take $\mathcal{L}_{x}=1 / 15$. As expected, the $\mathbb{P}_{0}$ and $\mathbb{P}_{5}^{\mathrm{WB}}$ scheme capture the steady solution up to machine precision. The $\mathbb{P}_{5}$ scheme gives an approximation of the solution, but remains far from the exact solution.

Table 4 reports the errors and CPU times for the three schemes under consideration. We draw the same conclusions as in section 6.1.2. In addition, we display the results of the $\mathbb{P}_{0}$ and $\mathbb{P}_{5}^{W B}$ schemes in figure 5 , for several physical times $(t=0 \mathrm{~s}, t=0.05 \mathrm{~s}$ and $t=5 \mathrm{~s})$. We observe that, starting with the same initial condition, we end up with the same steady state up to machine precision. However, the approximation of the transient state in the middle panel is much less diffusive when using the $\mathbb{P}_{5}^{\mathrm{WB}}$ scheme compared to the $\mathbb{P}_{0}$ scheme. This highlights the relevance of both the high-order accuracy and the well-balancedness property. 


\begin{tabular}{|c|c|c|c|c|c|c|c|}
\hline \multirow[b]{2}{*}{ scheme } & \multicolumn{3}{|c|}{$\psi_{x}^{f}$} & \multicolumn{3}{|c|}{$\|\boldsymbol{q}\|$} & \multirow[b]{2}{*}{ CPU time $(\mathrm{s})$} \\
\hline & $L^{1}$ & $L^{2}$ & $L^{\infty}$ & $L^{1}$ & $L^{2}$ & $L^{\infty}$ & \\
\hline $\mathbb{P}_{0}$ & $9.18 \mathrm{e}-15$ & $1.08 \mathrm{e}-14$ & $1.96 \mathrm{e}-14$ & $3.43 \mathrm{e}-15$ & $3.95 \mathrm{e}-15$ & $6.88 \mathrm{e}-15$ & 0.2991 \\
\hline $\mathbb{P}_{5}$ & $1.55 \mathrm{e}-03$ & $1.55 \mathrm{e}-03$ & $1.59 \mathrm{e}-03$ & $1.04 \mathrm{e}-03$ & $1.04 \mathrm{e}-03$ & $1.06 \mathrm{e}-03$ & 34.23 \\
\hline $\mathbb{P}_{5}^{\mathrm{WB}}$ & $6.53 \mathrm{e}-13$ & $8.38 \mathrm{e}-13$ & $1.44 \mathrm{e}-12$ & $3.38 \mathrm{e}-13$ & $3.82 \mathrm{e}-13$ & $5.47 \mathrm{e}-13$ & 4.708 \\
\hline
\end{tabular}

Table 4: Error on $\psi_{x}^{f}$, error on $\|\boldsymbol{q}\|$ and CPU time for perturbed steady state with friction, using each of the three schemes. Note that a steady solution is exactly captured after a transient state due to the propagation of the perturbation.
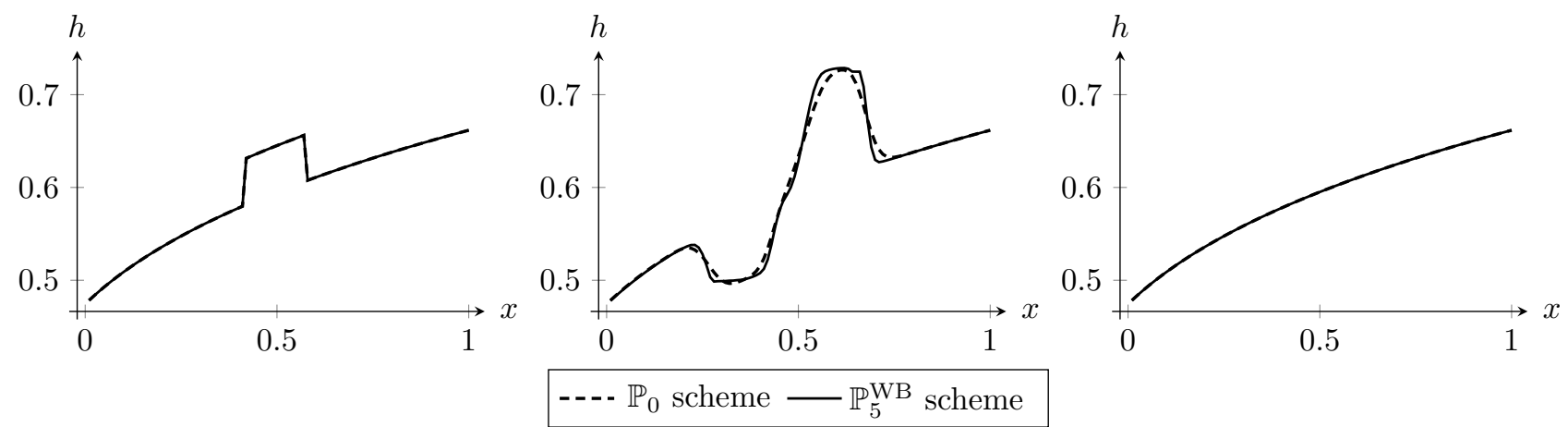

Figure 5: Water height for the perturbed steady state with friction, with the $\mathbb{P}_{0}$ scheme (dashed line) and the $\mathbb{P}_{5}^{\mathrm{WB}}$ scheme (solid line). Left panel: initial condition at $t=0 \mathrm{~s}$; middle panel: transient state at $t=0.05 \mathrm{~s}$; right panel: steady state at $t=5 \mathrm{~s}$.

\subsection{Order of accuracy assessment}

We now assess the accuracy of the high-order scheme. To that end, we propose two numerical experiments. The first one only involves the topography source term, while both topography and friction are considered in the second benchmark.

\subsubsection{Steady vortex}

This first experiment is a steady vortex (see $[52,29])$. On the space domain $(-1,1)^{2}$, we set the topography as $Z(x, y)=0.2 e^{0.5\left(1-r^{2}\right)}$, with $r^{2}=x^{2}+y^{2}$. We cancel the friction term by taking $k=0$. The exact solution, displayed in figure 6 , is then given by $W_{e x}=(h, h u, h v)^{\top}$, where we have set

$$
h(t, x, y)=1-\frac{1}{4 g} e^{2\left(1-r^{2}\right)}-Z(x, y) ; \quad u(t, x, y)=y e^{1-r^{2}} ; \quad v(t, x, y)=-x e^{1-r^{2}} .
$$

The initial conditions consist in computing a numerical average, with a quadrature formula of order $(d+1)$, of the exact solution in each cell. Similarly, the boundary conditions are obtained by evaluating the exact solution at the Gauss points on the domain boundary.

The simulations are carried out with the $\mathbb{P}_{3}^{\mathrm{WB}}$ and $\mathbb{P}_{5}^{\mathrm{WB}}$ schemes, until a final physical time $t_{\text {end }}=1 \mathrm{~s}$. In addition, we take $C=+\infty$ for each scheme. The results of the simulations are presented in figure 7 .

In all cases, the accuracy reaches the order $(d+1)$, as expected. Optimal accuracy is maintained thanks to the $u 2$ detection criteria. Indeed, on such smooth solutions, the DMP criterion (5.1) would wrongly lower the CPD in some cells by over-detecting smooth extrema. Here, the smoothness detector (5.4) is used to correct over-detection from the DMP criterion. The reader is referred to [29] for a comparison of the order with and without the $u 2$ criterion. In [29], the authors show that the $u 2$ criterion is mandatory to recover the expected 

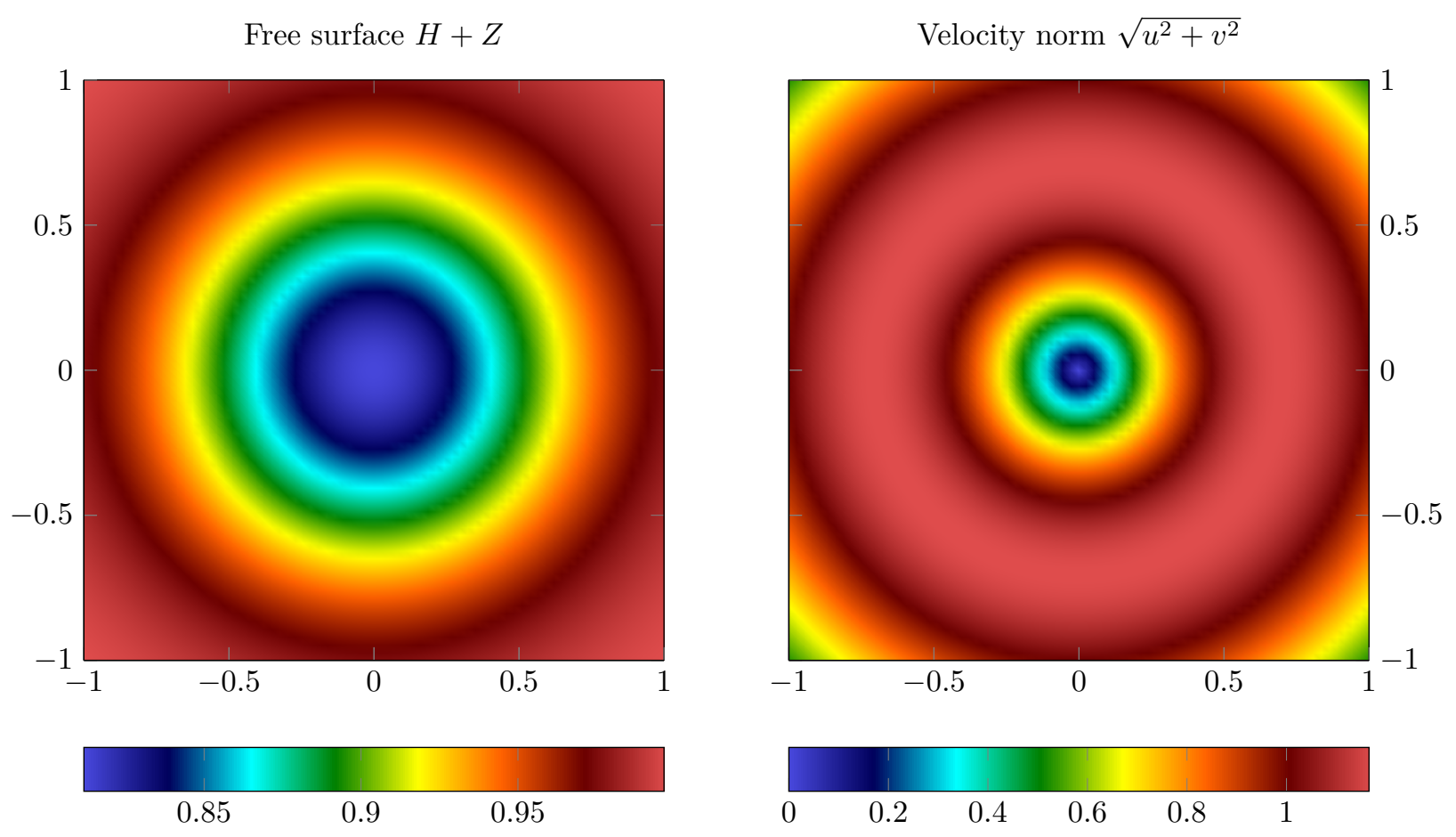

Figure 6: Exact solution for the steady vortex experiment. Left panel: free surface. Right panel: velocity norm (the vortex flows clockwise).

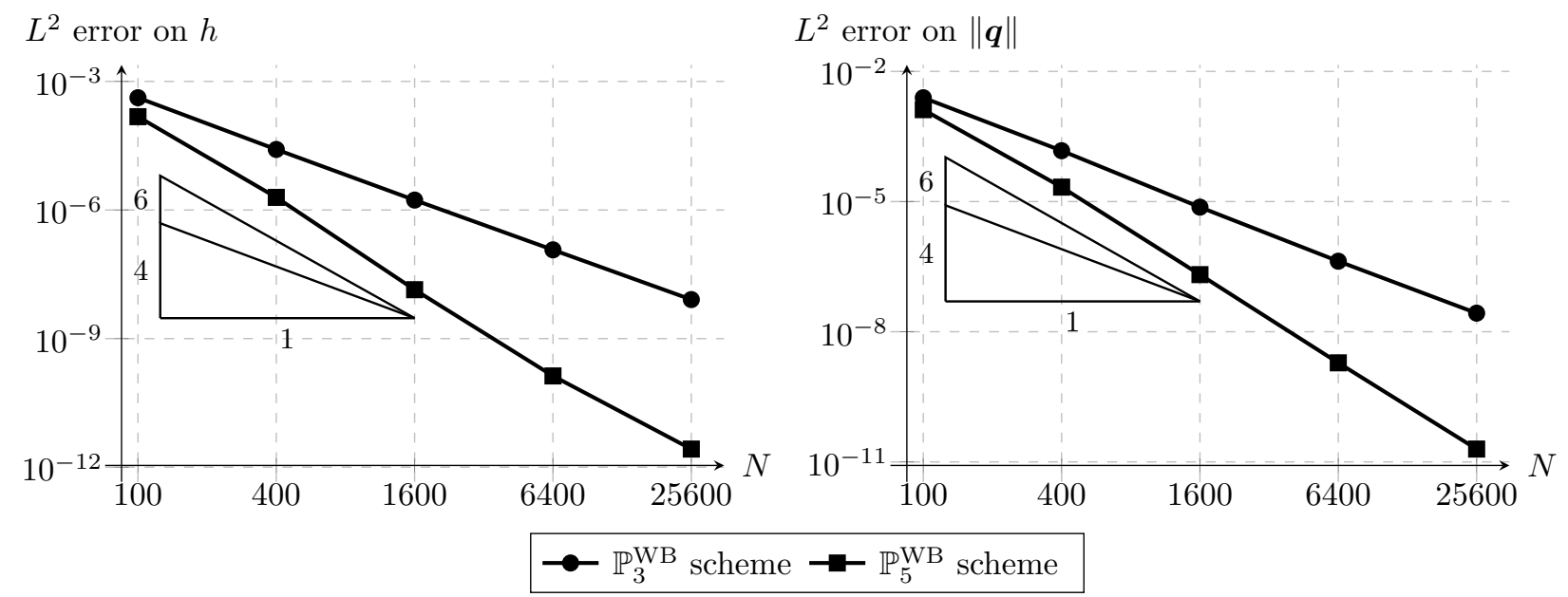

Figure 7: Error lines in $L^{2}$ norm for the exact solution with topography, using the $\mathbb{P}_{3}^{\mathrm{WB}}$ and $\mathbb{P}_{5}^{\mathrm{WB}}$ schemes. Left panel: $L^{2}$ error on $h$; right panel: $L^{2}$ error on $\|\boldsymbol{q}\|$.

order of accuracy.

\subsubsection{Experiment with topography and friction}

The second experiment for accuracy assessment concerns a two-dimensional steady state involving both the topography and the friction source term (see [53]). The exact solution is defined as follows. Let $\boldsymbol{r}=(x, y)^{\top}$. We 
assume, for this experiment, that $\|\boldsymbol{r}\| \neq 0$. The topography is given by:

$$
Z(x, y)=\frac{2 k\|\boldsymbol{r}\|-1}{2 g\|\boldsymbol{r}\|^{2}} .
$$

In addition, we set $W_{e x}=(h, \boldsymbol{q})^{\top}$, where

$$
h(t, x, y)=1 \quad \text { and } \quad \boldsymbol{q}(t, x, y)=\frac{\boldsymbol{r}}{\|\boldsymbol{r}\|^{2}} .
$$

For the purpose of the simulation, we consider the exact solution on the space domain $(0.4,1)^{2}$, with a Manning coefficient $k=1$. As in the previous experiment, the initial and boundary conditions derive from the exact solution.

In order to check the high-order accuracy of the schemes, the benchmark is carried out with the $\mathbb{P}_{3}^{\mathrm{WB}}$ and $\mathbb{P}_{5}^{\mathrm{WB}}$ schemes. The final physical time is $t_{\mathrm{end}}=0.1 \mathrm{~s}$, and we take once again $C=+\infty$. Convergence curves are presented in figure 8.

$L^{2}$ error on $h$

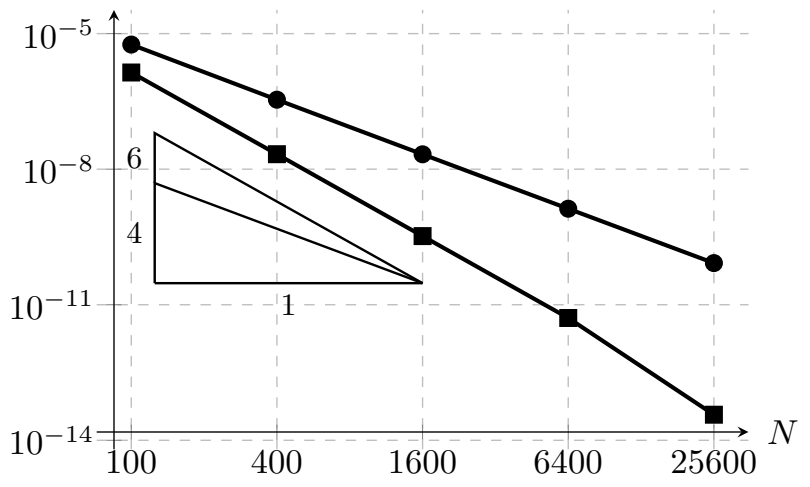

$L^{2}$ error on $\|\boldsymbol{q}\|$

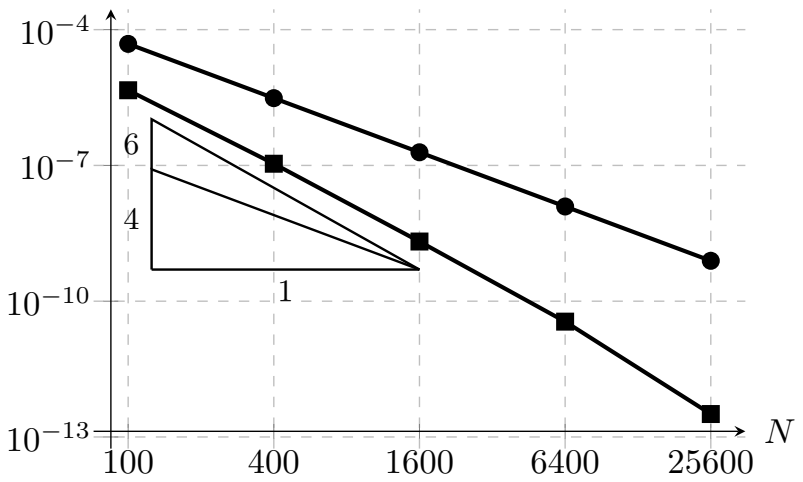

$$
\rightarrow \mathbb{P}_{3}^{\mathrm{WB}} \text { scheme } \rightarrow-\mathbb{P}_{5}^{\mathrm{WB}} \text { scheme }
$$

Figure 8: Error lines in $L^{2}$ norm for the exact solution with topography and friction, using the $\mathbb{P}_{3}^{\mathrm{WB}}$ and $\mathbb{P}_{5}^{\mathrm{WB}}$ schemes. Left panel: $L^{2}$ error on $h$; right panel: $L^{2}$ error on $\|\boldsymbol{q}\|$.

Once again, we obtain the expected order of accuracy, around $d+1$. Similarly to the previous experiment, this order of accuracy is recovered thanks to the $u 2$ criterion in addition to the DMP criterion.

\subsection{Dam break test cases}

This section is dedicated to the validation of dam-break benchmarks. We first consider a dam break over a dry bottom in one space direction. Such a simulation will highlight the relevance of the well-balancedness correction and the MOOD procedure. Next, we present a two-dimensional partial dam break.

\subsubsection{One-dimensional dry dam break}

We consider the academic square domain $[0,1]^{2}$, and the topography is given by:

$$
Z(x, y)=\frac{e^{x}}{e^{1}}-e^{-1},
$$


such that $Z(0, y)=0$ and $Z(1, y)=1$. The initial free surface consists in a double dam break, obtained by setting:

$$
h(0, x, y)+Z(x, y)= \begin{cases}2 & \text { if } x<\frac{1}{2} \\ Z(x, y) & \text { otherwise }\end{cases}
$$

In addition, the initial discharge is zero, i.e. $\boldsymbol{q}(0, x, y)=\mathbf{0}$. The Manning coefficient is set to 1 and the boundaries are considered to be solid walls. The experiment is carried out with the $\mathbb{P}_{0}$ and $\mathbb{P}_{5}^{\mathrm{WB}}$ schemes. The final physical time is $t_{\text {end }}=0.07 \mathrm{~s}$, and we set $C=+\infty$. We also take $\mathcal{L}_{x}=0.1$. The results are presented in Figures 9 and 10 .

Figure 9 shows a comparison between the free surface obtained with the $\mathbb{P}_{0}$ scheme and the one obtained with the $\mathbb{P}_{5}^{\mathrm{WB}}$ scheme, using $50=25 \times 2$ cells in each case. We also display a reference solution, obtained by using the $\mathbb{P}_{0}$ scheme with $1000=500 \times 2$ discretization cells.

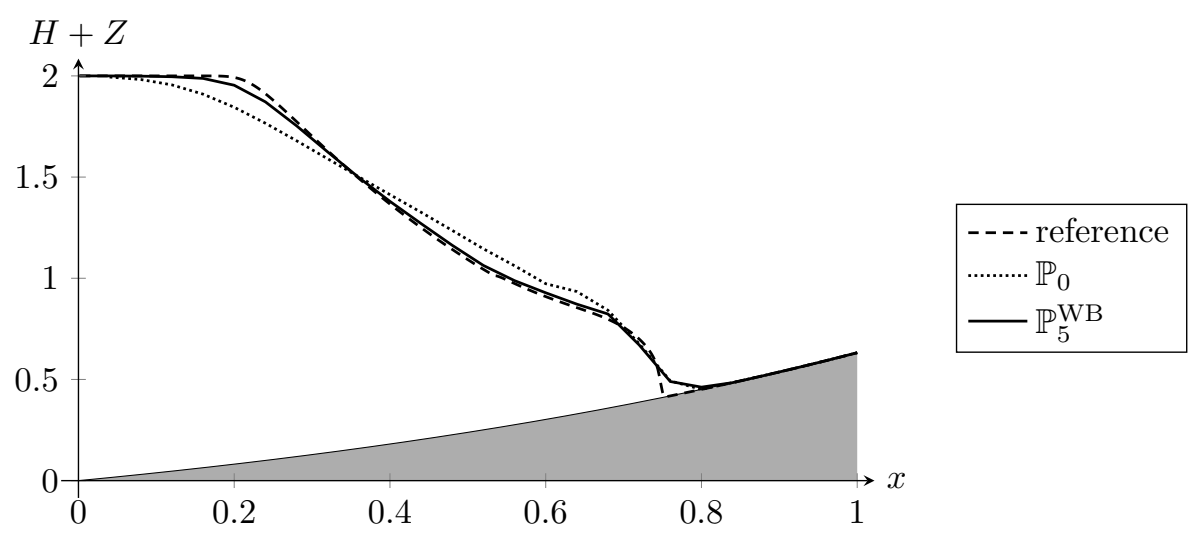

Figure 9: Free surface for the dam-break on a dry slope experiment; reference solution (dashed line), $\mathbb{P}_{0}$ scheme (dotted line), $\mathbb{P}_{5}^{\mathrm{WB}}$ scheme (solid line). The gray area represents the topography.

Figure 9 highlights the relevance of the well-balancedness property as well as the high-order accuracy. First, despite the coarse grid, the results from the $\mathbb{P}_{5}^{\mathrm{WB}}$ scheme are close to the reference solution, except in one cell close to the dry/wet transition, where the PAD detector is activated. In addition, we note that the free surface is unperturbed close to the left edge of the domain. Indeed, the waves from the dam break have not yet reached the edges of the domain at $t=t_{\text {end }}$, and the area located in the vicinity of the left edge is in a lake at rest configuration. This essential property exactly holds for the $\mathbb{P}_{5}^{\mathrm{WB}}$ scheme. This behavior is obtained thanks to the well-balancedness correction, which forces the well-balanced scheme to be activated in lake at rest-type situations.

In figure 10, the well-balancedness coefficient in the $x$-direction $\theta_{x}$ is depicted, together with the free surface and the topography, for $t=t_{\text {end }} / 2$ and $t=t_{\text {end }}$, for the $\mathbb{P}_{5}^{\mathrm{WB}}$ scheme.

In the left panel of figure 10, we observe that $\theta_{x}$ is zero in areas that have not yet been impacted by the waves, i.e. in the areas where a lake at rest configuration is found. As a consequence, in these areas (close to the edges of the domain), the well-balanced scheme is used. Similar conclusions are drawn from the right panel of figure 10. The edges of the domain are still considered to be at rest, which is evidenced by the convex combination parameter being very close to zero.

\subsubsection{Two-dimensional partial dam-break}

The second experiment concerns a two-dimensional partial dam break (see [55, 29, 53]). An extensive study of this experiment, focusing on the differences between various reconstruction degrees and MOOD criteria, has been 


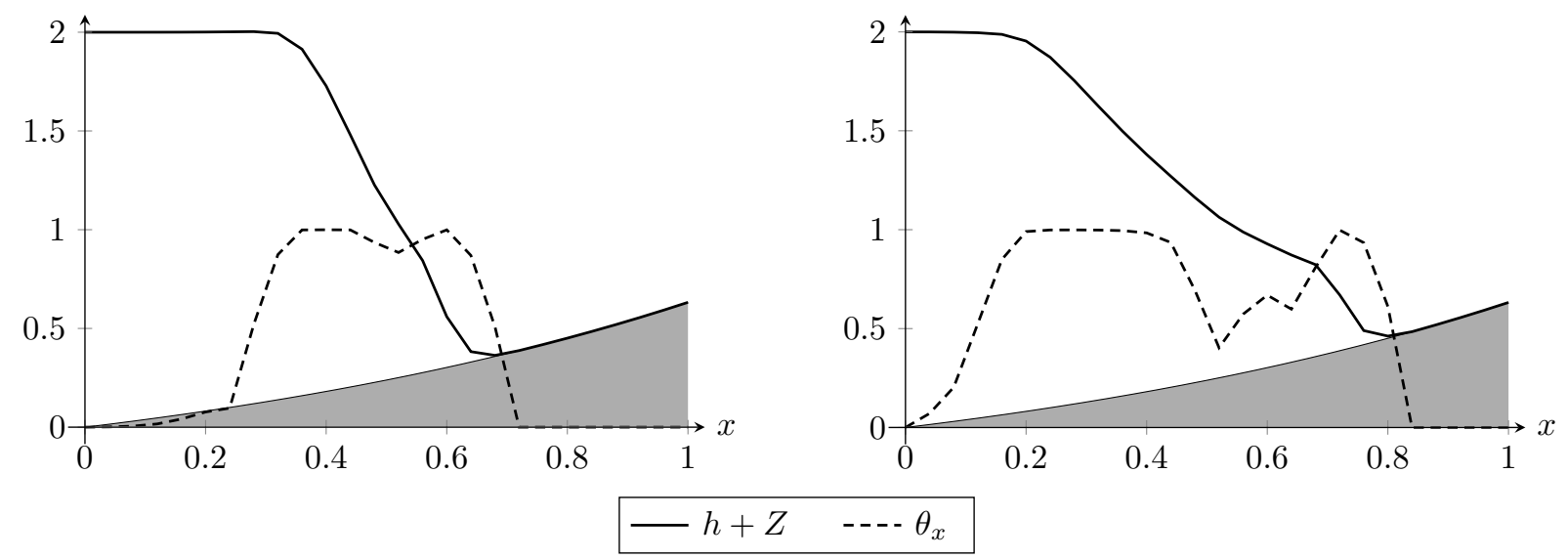

Figure 10: Free surface $h+Z$ (solid line) and well-balancedness parameter in the $x$-direction $\theta_{x}$ (dashed line) for the dam-break on a dry slope experiment using the $\mathbb{P}_{5}^{\mathrm{WB}}$ scheme. The gray area represents the topography. Left panel: $t=t_{\text {end }} / 2$; right panel: $t=t_{\text {end }}$.

carried out in [29]. In [29], the authors show that the depth of the vortices appearing at the edges of the dam strongly depends on the degree of the reconstruction and the MOOD criteria used. However, in [29], the friction source term was not present, and the authors only studied the effects of the topography. Thus, in the present paper, we focus on the impact of the friction source term, by carrying out the simulation with three different Manning coefficients.

For this experiment, the space domain is $[-100,100] \times[-100,100]$, and the topography is given as follows:

$$
Z(x, y)= \begin{cases}1 & \text { if } x \leq-5, \\ 0 & \text { if } x \geq 5 \\ 0.1(5-x) & \text { if }-5<x<5 \text { and }-40<y<40 \\ 12 & \text { if }-5<x<5 \text { and } y \in[-100,-40] \cup[40,100] .\end{cases}
$$

It represents a 12 meters high, 10 meters wide broken dam. Initially, the reservoir (to the left) is filled, as follows:

$$
h(0, x, y)= \begin{cases}10-Z(x, y) & \text { if } x \leq-5, \\ 5-Z(x, y) & \text { if } x \geq 5, \\ 5-Z(x, y) & \text { if }-5<x<5 \text { and }-40<y<40 \\ 0 & \text { if }-5<x<5 \text { and } y \in[-100,-40] \cup[40,100] .\end{cases}
$$

The water is initially at rest, i.e. $\boldsymbol{q}(0, x, y)=\mathbf{0}$. For this simulation, we use wall boundary conditions. All the simulations are carried out with $40000=200 \times 200$ discretization cells.

The goal of this simulation is to compare the results from the $\mathbb{P}_{0}, \mathbb{P}_{1}^{\mathrm{WB}}$ and $\mathbb{P}_{5}^{\mathrm{WB}}$ schemes. Moreover, the simulation is carried out with various Manning coefficients, namely $k=0, k=0.25$ and $k=2$, and until the final physical time $t_{\text {end }}=7 \mathrm{~s}$. In addition, we set $C=0.5$.

The results of the simulations are displayed in figure $11(k=0)$, in figure $12(k=0.25)$ and in figure 13 $(k=2)$. We use the same color scale in all figures.

Figure 11 and figure 12 show that the shock wave to the right of the dam and the rarefaction wave to the left of the dam are clearly more smeared when using the $\mathbb{P}_{0}$ scheme instead of the $\mathbb{P}_{1}^{\mathrm{WB}}$ or the $\mathbb{P}_{5}^{\mathrm{WB}}$ scheme. In 

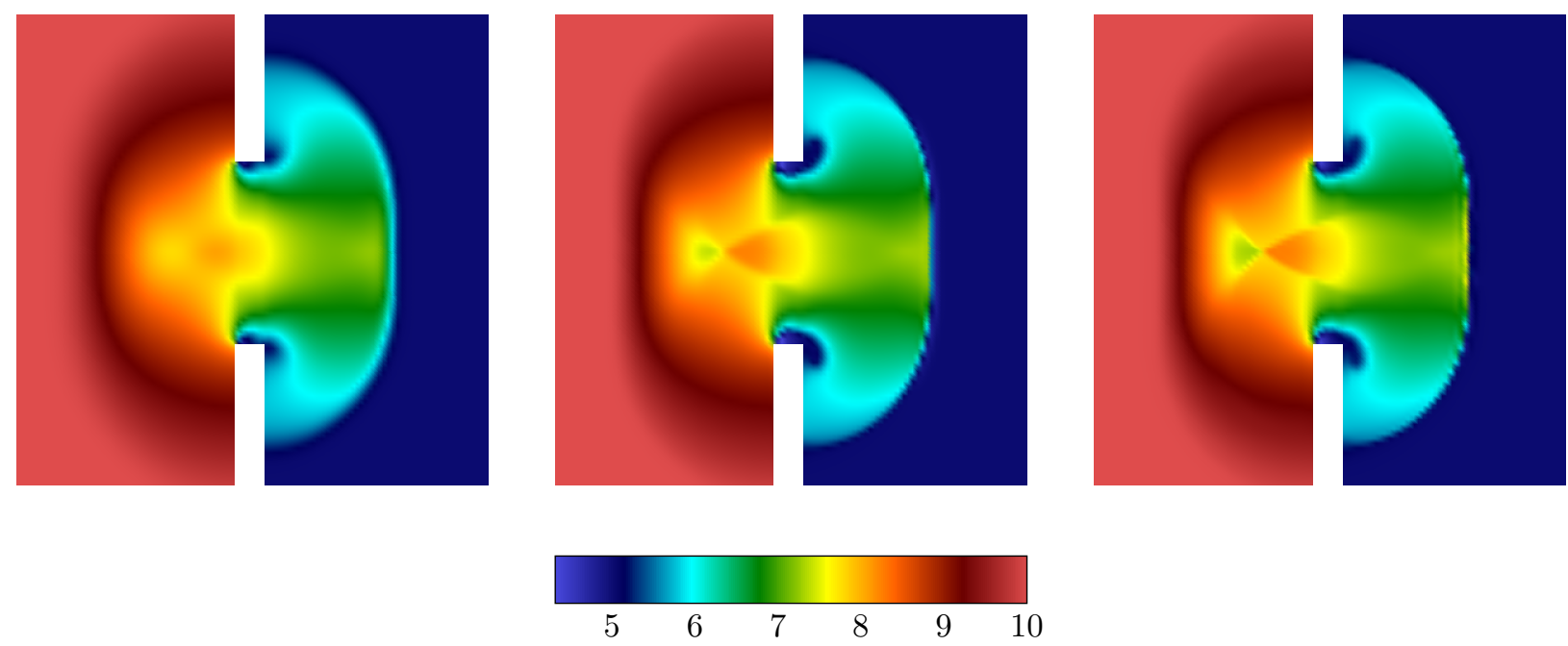

Figure 11: Free surface for the partial dam-break simulation with $k=0$. From left to right: results of the $\mathbb{P}_{0}, \mathbb{P}_{1}^{\mathrm{WB}}$ and $\mathbb{P}_{5}^{\mathrm{WB}}$ schemes.
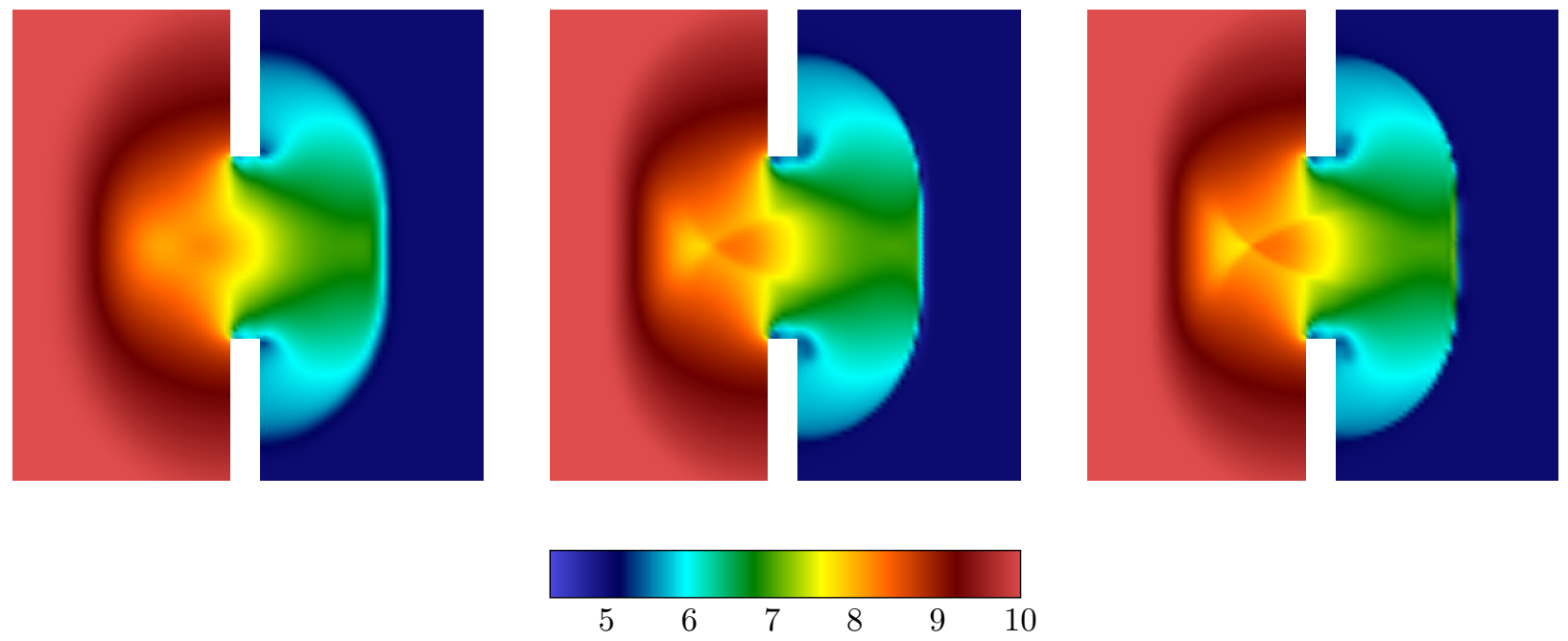

Figure 12: Free surface for the partial dam-break simulation with $k=0.25$. From left to right: results of the $\mathbb{P}_{0}, \mathbb{P}_{1}^{\mathrm{WB}}$ and $\mathbb{P}_{5}^{\mathrm{WB}}$ schemes.

addition, the shock structure at the center of the water flow is not visible with the $\mathbb{P}_{0}$ scheme. This structure, although smeared, is visible with the $\mathbb{P}_{1}^{\mathrm{WB}}$ scheme, and turns out to be very well defined with the $\mathbb{P}_{5}^{\mathrm{WB}}$ scheme. We draw similar conclusions from figure 13. The smearing of the shock wave and the rarefaction wave is noticeable with the first- and second-order schemes, but it is strongly reduced with the high-order scheme. In addition, the important friction has caused the central structure to nearly disappear.

An important remark we make here concerns the vortices present at the edges of the dam in Figures 11 and 12. The presence of the friction source term dampens the depth, as well as the size, of these vortices. We focus on the top vortex, whose characteristics are similar to the bottom one since the experiment is symmetric with respect to the $y=0$ line. This behavior is displayed in table 5 , where the approximate size and the depth of the vortex are collected.

Concerning the left rarefaction wave, the relevant indicators are the position of the head of the rarefaction 

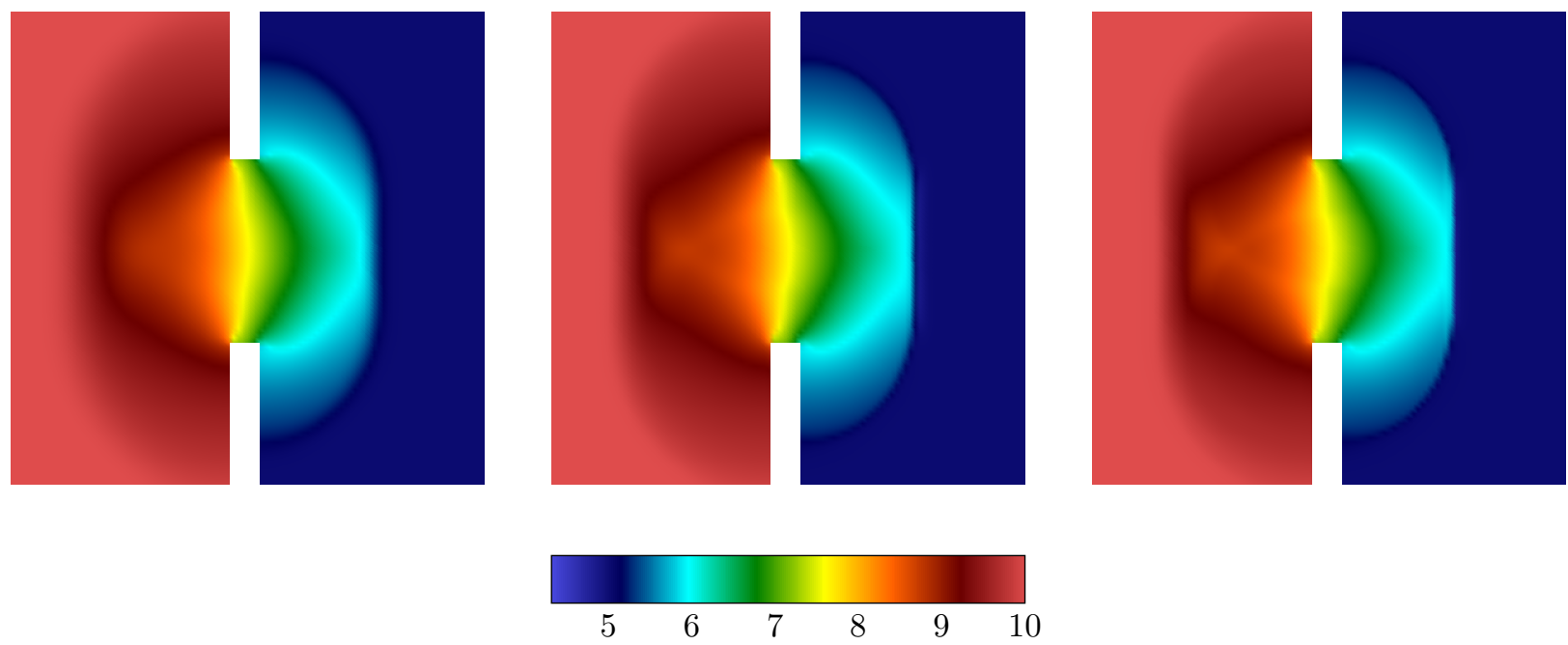

Figure 13: Free surface for the partial dam-break simulation with $k=2$. From left to right: results of the $\mathbb{P}_{0}, \mathbb{P}_{1}^{\mathrm{WB}}$ and $\mathbb{P}_{5}^{\mathrm{WB}}$ schemes.

\begin{tabular}{ccc}
\hline Manning coefficient & Vortex size & Vortex depth \\
\hline$k=0$ & $84 \mathrm{~m}^{2}$ & $4.28 \mathrm{~m}$ \\
\hline$k=0.25$ & $17 \mathrm{~m}^{2}$ & $5.45 \mathrm{~m}$ \\
\hline$k=2$ & $0 \mathrm{~m}^{2}$ & $7.23 \mathrm{~m}$ \\
\hline
\end{tabular}

Table 5: Depth and approximate size of the deepest vortex, for the $\mathbb{P}_{5}^{\mathrm{WB}}$ scheme. For the case where $k=2$, there is no vortex, and the table displays the free surface at the point where the vortex would be located if the Manning coefficient were lower.

wave, its size, and its amplitude along the $y=0$ line. Those quantities are reported in table 6 , where we chose to compute the amplitude of the rarefaction wave by subtracting the water height at the tail from the water height at the head.

\begin{tabular}{cccc}
\hline Manning coefficient & Size & Amplitude & Head \\
\hline$k=0$ & $39 \mathrm{~m}$ & $2.68 \mathrm{~m}$ & $x=-74 \mathrm{~m}$ \\
\hline$k=0.25$ & $38 \mathrm{~m}$ & $2.28 \mathrm{~m}$ & $x=-74 \mathrm{~m}$ \\
\hline$k=2$ & $31 \mathrm{~m}$ & $1.29 \mathrm{~m}$ & $x=-74 \mathrm{~m}$ \\
\hline
\end{tabular}

Table 6: Left rarefaction wave: approximate size, water height amplitude and position of the head, with respect to the Manning coefficient.

Concerning the shock wave, we report on its position and its amplitude along the line $y=0$, given in table 7 . Similarly to the rarefaction wave, the amplitude of the shock wave is obtained by computing the difference between the water height to the left of the wave and the water height to its right. Note that, since those computations are performed on the numerical results of the $\mathbb{P}_{5}^{\mathrm{WB}}$ scheme, the shock wave takes only a couple of cells, and the evaluation of its position is fairly accurate. In addition, the amplitude of the shock wave presented for $k=0$ in table 7 is very similar to the results obtained in [29], although the authors do not use the same scheme.

Tables 6 and 7 give evidence about the effect of the friction on the water flow. The Manning term dampens the amplitudes of both the rarefaction wave and the shock wave, while an increase in the friction coefficient is accompanied by a diminution of the size of the rarefaction wave, and a decrease in the distance traveled by the 


\begin{tabular}{ccc}
\hline Manning coefficient & Position & Amplitude \\
\hline$k=0$ & $x=60 \mathrm{~m}$ & $2.28 \mathrm{~m}$ \\
\hline$k=0.25$ & $x=58 \mathrm{~m}$ & $1.96 \mathrm{~m}$ \\
\hline$k=2$ & $x=53 \mathrm{~m}$ & $0.98 \mathrm{~m}$ \\
\hline
\end{tabular}

Table 7: Right shock wave: approximate position and water height amplitude, with respect to the Manning coefficient.

shock wave. This behavior is expected, as an increase in friction leads to a decrease in discharge, as evidenced by the expression (2.23). The discharge decrease leads to a slower travel time of the shock wave, which means that the wave will travel less distance.

Finally, we observe from table 6 that the friction does not change the position of the head of the rarefaction wave. This behavior is also expected from the expression of the friction source term given by (1.8) in 1D and (1.1) in 2D. Near the head of the rarefaction wave, the water is almost at rest, since no wave has already perturbed the initial rest condition, leading to a negligible impact of the friction source term, which means the head of the rarefaction wave travels at the same speed for $k=0, k=0.25$ or $k=2$. Therefore, the value of the Manning coefficient does not alter the position of the head of the rarefaction wave.

\subsection{Simulation of the 2011 Töhoku tsunami}

We tackle the simulation of the tsunami that hit the Japanese region of Tōhoku in 2011. This simulation is based on real data, see for instance [30]. This real data consists in a uniform Cartesian mesh made of about 13 million rectangles, where the cell topography and the initial free surface for the tsunami simulation are given, see figure 14. The initial discharge is set to zero, that is $\boldsymbol{q}(0, x, y)=\mathbf{0}$. Homogeneous Neumann boundary conditions are prescribed at each boundary. In addition, we set $k=0.05$.

To assess the simulation accuracy, we compare real physical measurements from DART buoys far from the Japanese coast with the numerical approximation. The positions of these sensors are depicted in the left panel of figure 15. The sensors have measured the water height during one hour, and thus we set the final physical time to $t_{\text {end }}=3600 \mathrm{~s}$. Note that, even though the mesh contains a large number of rectangles, the side length of each cell is close to $900 \mathrm{~m}$. Such large cells create large topography gradients with respect to the characteristic cell size, especially close to the Kuril trench the tsunami originated from. For instance, the right panel of figure 15 shows the topography over the solid horizontal line in the left panel of figure 15. The extreme topography gradients, especially around the Kuril trench, have to be correctly handled by the scheme, which is the main difficulty of this simulation.

This presence of large topography gradients also highlights why using a well-balanced scheme is absolutely necessary in such simulations. In figure 16, we display the first time iteration of the standard HLL scheme from [48] applied to the tsunami simulation. We observe large oscillations, which completely destroy the numerical approximation, even at this first time step. Therefore, using a well-balanced scheme is indeed crucial for such simulations. Such issues may be less prominent when dealing with finer meshes to approximate coastal areas, like in $[25,58,2]$ and references therein.

We check the numerical results of the $\mathbb{P}_{0}, \mathbb{P}_{1}^{\mathrm{WB}}$ and $\mathbb{P}_{3}^{\mathrm{WB}}$ schemes. We ran the simulation until the final time $t_{\text {end }}=1 \mathrm{~h}$ on 48 computational cores; the $\mathbb{P}_{0}$ scheme took around 1 hour of CPU time, whereas the $\mathbb{P}_{1}^{\mathrm{WB}}$ and $\mathbb{P}_{3}^{W B}$ schemes took respectively around $2.5 \mathrm{~h}$ and around $10 \mathrm{~h}$. The numerical results are displayed in figure 17. The $\mathbb{P}_{0}$ and $\mathbb{P}_{1}^{W B}$ schemes yield comparable results, and the second-order result is, as expected, less diffusive than 

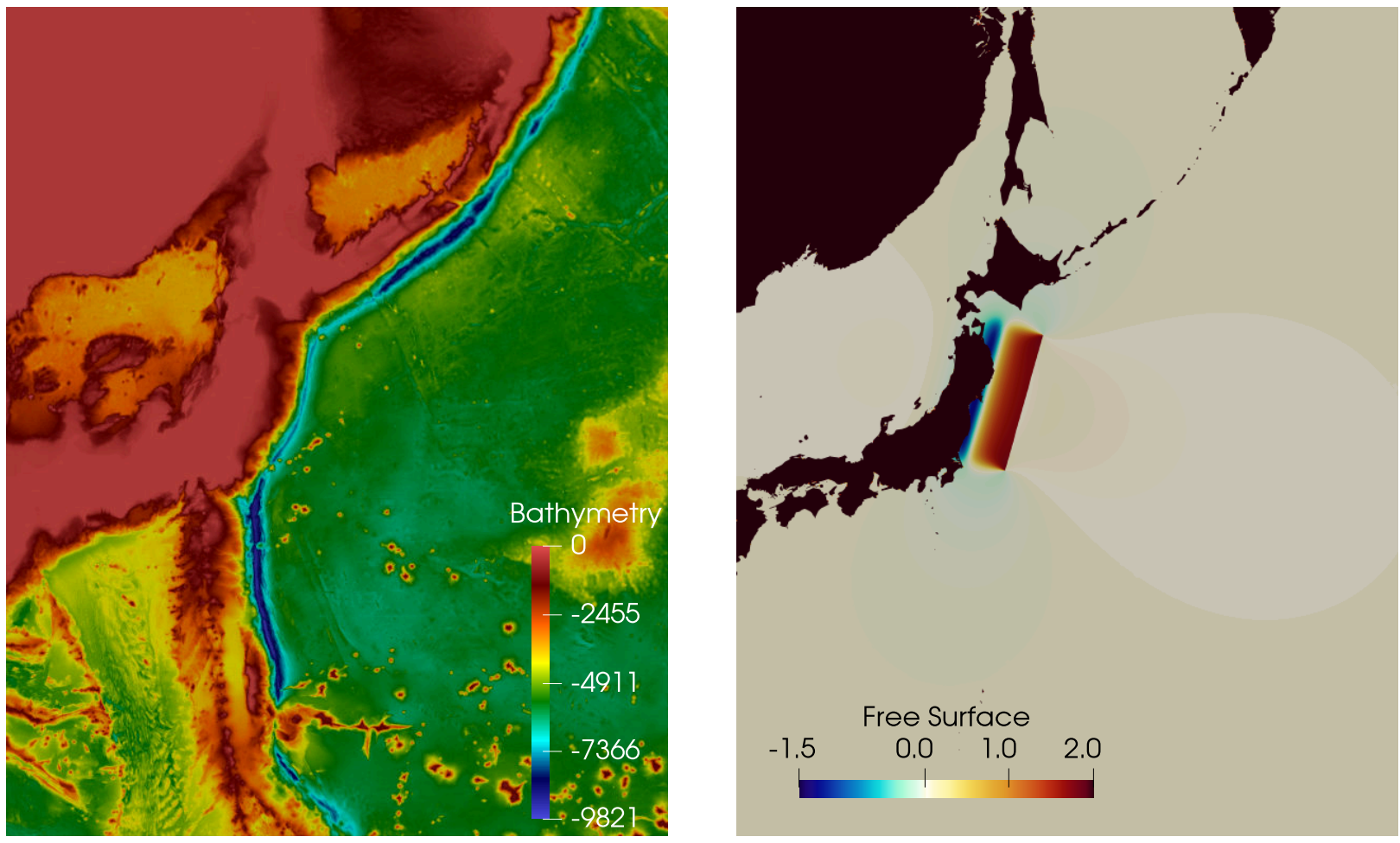

Figure 14: Depiction of the 2011 tsunami simulation. Left panel: bathymetry (submerged topography). The Kuril trench is the deepest part of the ocean, depicted in deep blue. The continents are represented in red. Right panel: initial free surface. The continents are depicted in black, the average water surface in gray, and the initial tsunami wave lies over the Kuril trench, next to the Japanese coast.
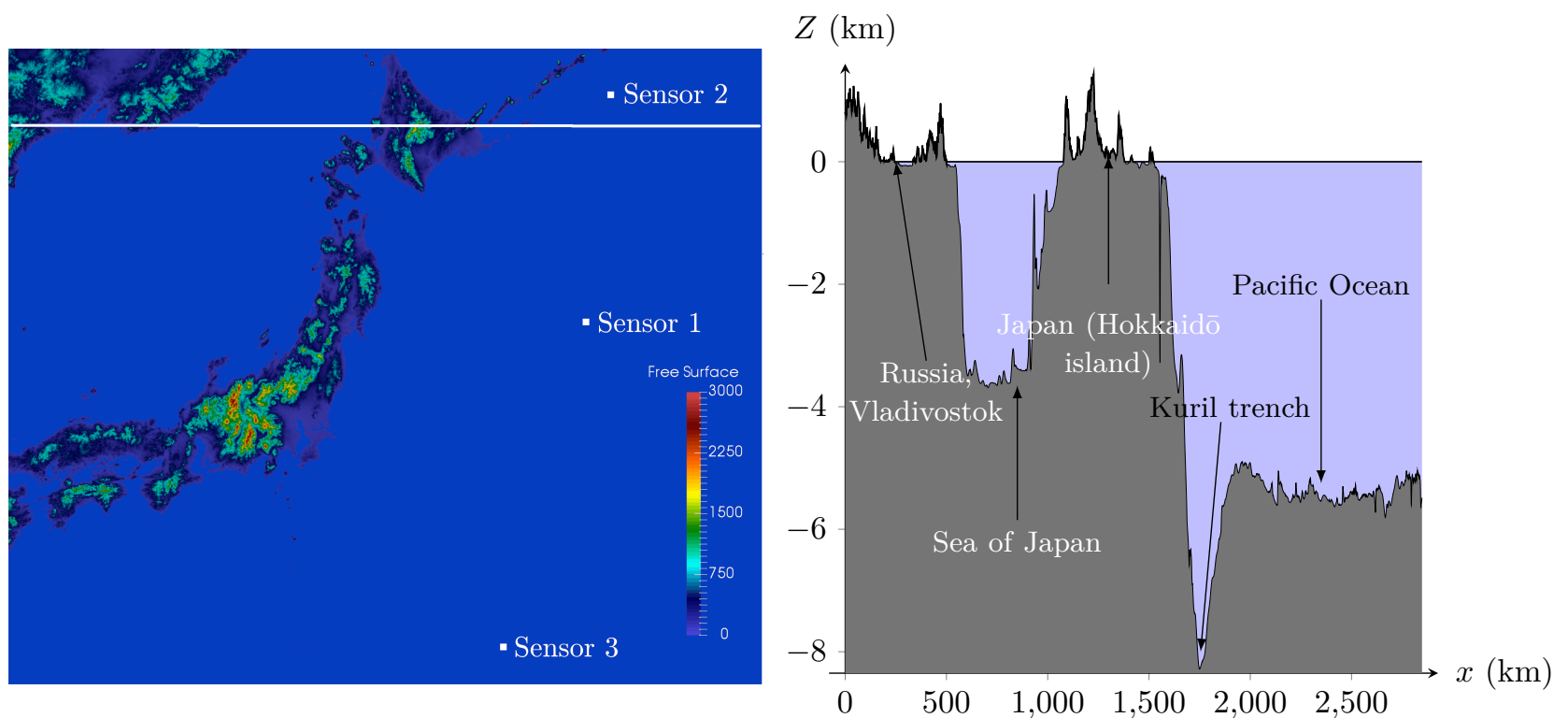

Figure 15: Left panel: position of the three sensors. Right panel: depiction of the topography over the white line drawn in the left panel. 


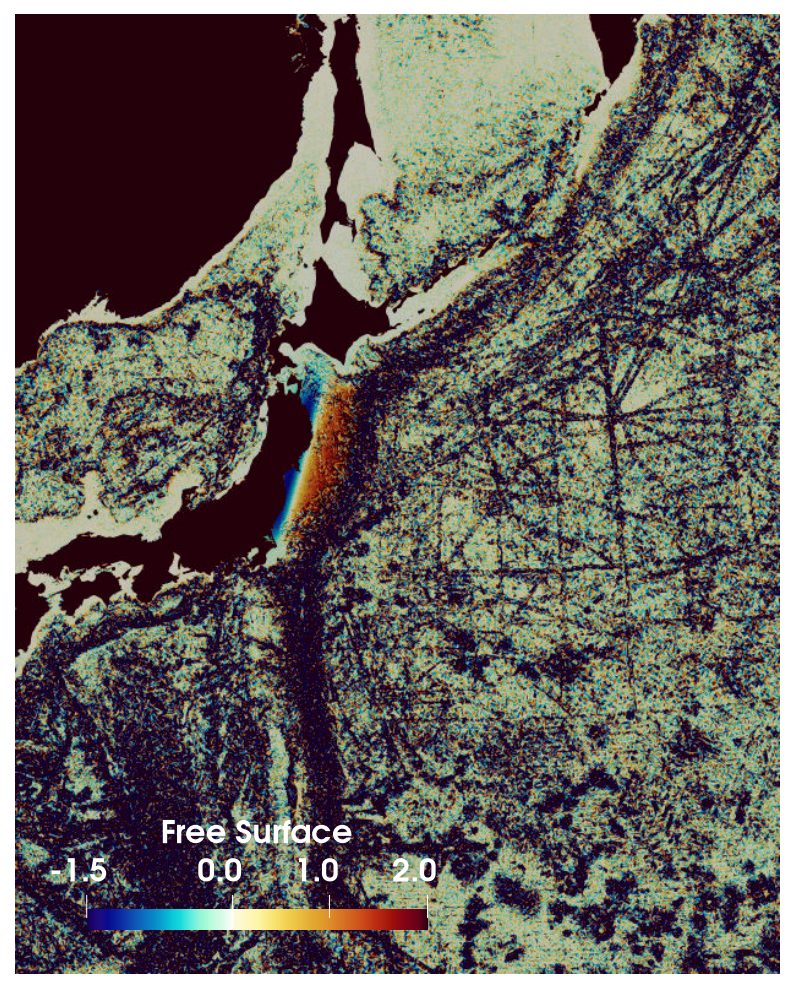

Figure 16: First time iteration of the 2011 Tōhoku tsunami simulation using a non-well-balanced scheme. The average sea surface height is represented in gray, and the continents are displayed in dark red. Oscillations, which correspond to the regions with a large bathymetry gradient displayed in the left panel of figure 14, are clearly visible. Note that the color scheme is the same as in the right panel of figure 14, which emphasizes that the oscillations are much larger than the tsunami.

the first-order one, with much more structure present within the waves. Unfortunately, the results of the $\mathbb{P}_{3}^{\text {WB }}$ scheme are unsatisfactory. The extreme topography gradients present in the domain, like the ones depicted in the right panel of figure 15, have led to an over-limitation of the MOOD method to remove the spurious oscillations, and the fourth-order solution ends up looking very similar to the first-order one.

In figure 18, we display the sea surface height ( $\mathrm{SSH})$, that is to say the difference between the water height and the average surface elevation, at each of the three sensors. The physical data is compared to the results from the $\mathbb{P}_{0}$ and $\mathbb{P}_{1}^{W B}$ schemes. We observe that, although the $\mathbb{P}_{0}$ scheme already gives a good approximation of the data, the $\mathbb{P}_{1}^{\mathrm{WB}}$ approximation is even better. Namely, the correct tsunami propagation time is captured, and the well-balancedness of the schemes ensure that no spurious oscillations come from the balance between flux and topography. This result questions the need to even use higher-order schemes for this simulation with such large cells, since the second-order results are already very close to the physical data.

\section{Acknowledgments}

V. Michel-Dansac extends his thanks to the Service Hydrographique et Océanographique de la Marine (SHOM) for financial support. C. Berthon and F. Foucher would like to thank the project MUFFIN ANR-19-CE46-0004 for financial support. S. Clain acknowledges the financial support by FEDER - Fundo Europeu de Desenvolvimento Regional, Portugal, through COMPETE 2020 - Programa Operational Fatores de Competitividade, and the National Funds through FCT - Fundação para a Ciência e a Tecnologia, Portugal, project No. UID/FIS/04650/2013 and project No. POCI-01-0145-FEDER-028118. 

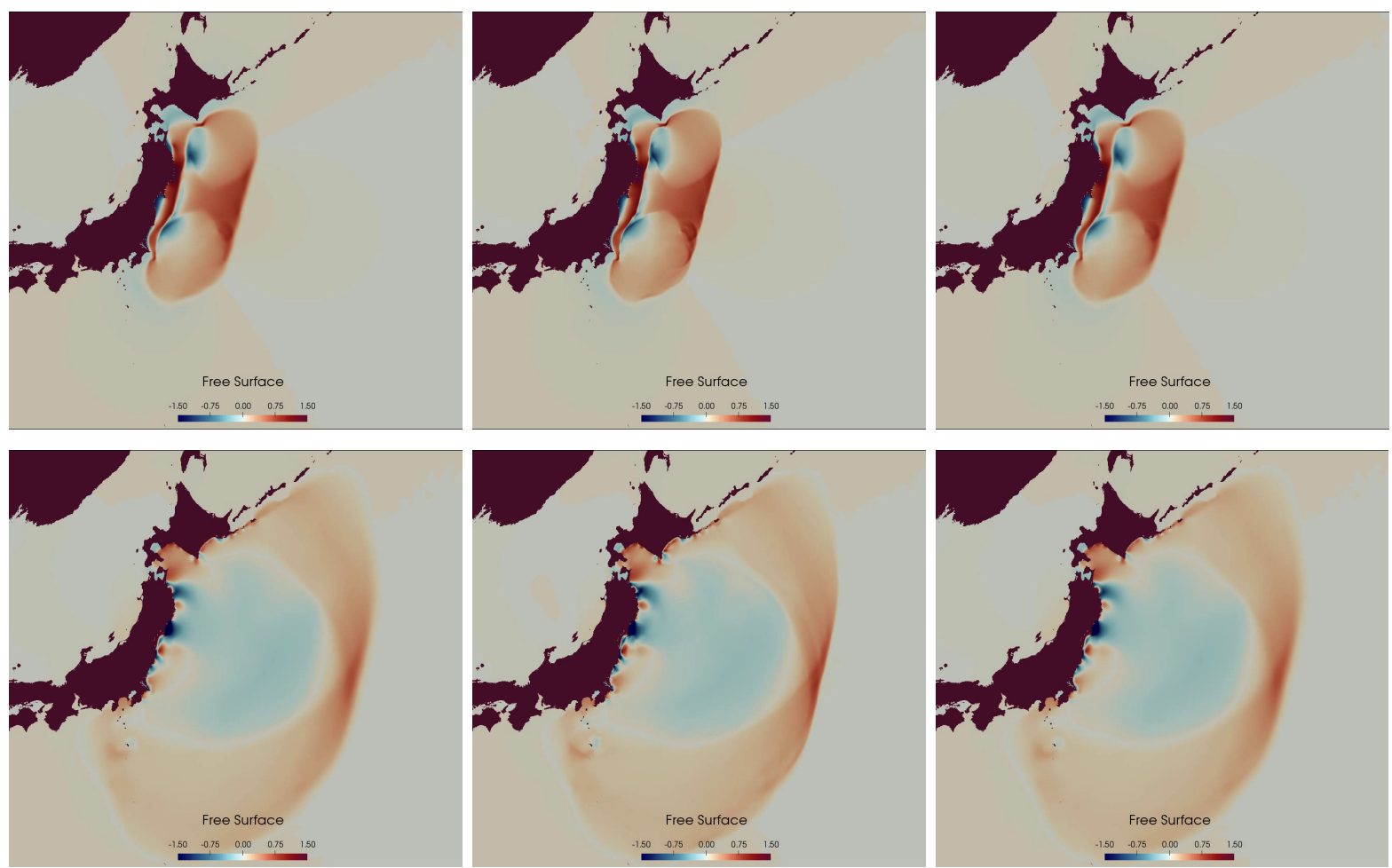

Figure 17: Simulation of the 2011 Töhoku tsunami with the $\mathbb{P}_{0}$ scheme (left panels), the $\mathbb{P}_{1}^{\mathrm{WB}}$ scheme (middle panels) and the $\mathbb{P}_{3}^{\mathrm{WB}}$ scheme (right panels). Snapshots taken at times $t=720 \mathrm{~s}$ (top panels) and $t=3600 \mathrm{~s}$ (bottom panels). The average sea surface height is represented in gray, and the continents are displayed in dark red.

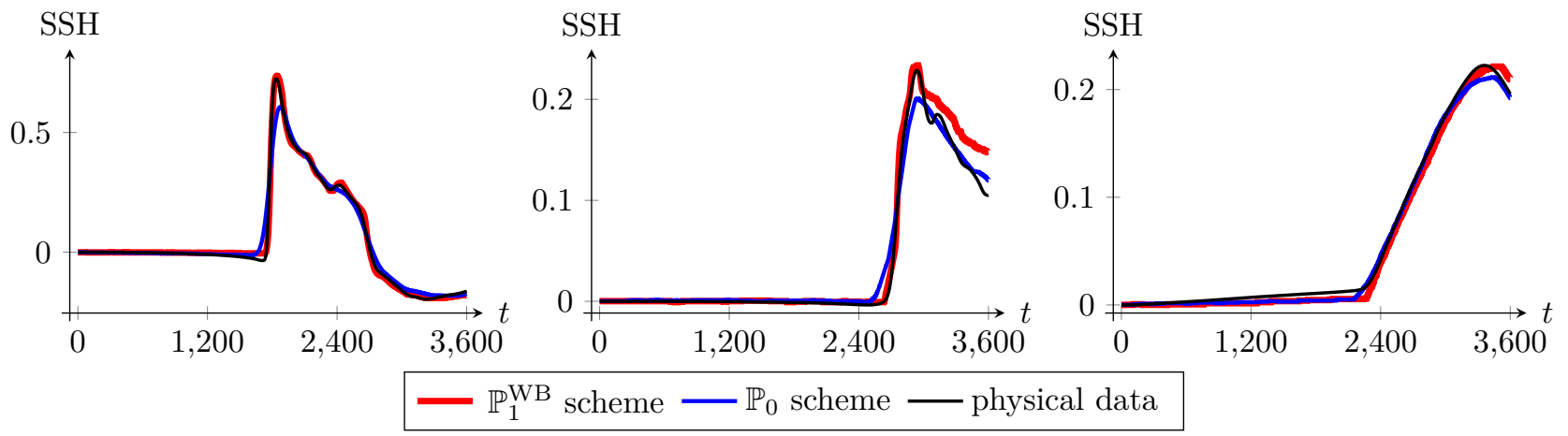

Figure 18: Sea surface height at each sensor (from left to right, sensor \#1 to sensor \#3, whose positions are displayed in figure 15). The physical data is represented in black, the $\mathbb{P}_{0}$ approximation in blue, and the $\mathbb{P}_{1}^{\mathrm{WB}}$ approximation in red. The total water depths below sensors $\# 1, \# 2$ and $\# 3$ are respectively $5700 \mathrm{~m}, 6600 \mathrm{~m}$ and $4400 \mathrm{~m}$.

\section{References}

[1] M. Abramowitz and I. A. Stegun, editors. Handbook of mathematical functions with formulas, graphs, and mathematical tables. Dover Publications, Inc., New York, 1992. Reprint of the 1972 edition.

[2] L. Arpaia and M. Ricchiuto. Well balanced residual distribution for the ALE spherical shallow water equations on moving adaptive meshes. J. Comput. Phys., 405:109173, 2020. 
[3] E. Audusse, F. Bouchut, M.-O. Bristeau, R. Klein, and B. Perthame. A fast and stable well-balanced scheme with hydrostatic reconstruction for shallow water flows. SIAM J. Sci. Comput., 25(6):2050-2065, 2004.

[4] E. Audusse and M.-O. Bristeau. A well-balanced positivity preserving "second-order" scheme for shallow water flows on unstructured meshes. J. Comput. Phys., 206(1):311-333, 2005.

[5] D. S. Balsara. Self-adjusting, positivity preserving high order schemes for hydrodynamics and magnetohydrodynamics. J. Comput. Phys., 231(22):7504-7517, 2012.

[6] J. P. Berberich, P. Chandrashekar, and C. Klingenberg. A General Well-Balanced Finite Volume Scheme for Euler Equations with Gravity. In Theory, Numerics and Applications of Hyperbolic Problems I, pages 151-163. Springer International Publishing, 2018.

[7] A. Bermudez and M. E. Vazquez. Upwind methods for hyperbolic conservation laws with source terms. Comput. \& Fluids, 23(8):1049-1071, 1994.

[8] C. Berthon and C. Chalons. A fully well-balanced, positive and entropy-satisfying Godunov-type method for the shallow-water equations. Math. Comp., 85(299):1281-1307, 2016.

[9] C. Berthon and V. Desveaux. An entropy preserving MOOD scheme for the Euler equations. Int. J. Finite Vol., 11, 2014.

[10] C. Berthon and F. Foucher. Efficient well-balanced hydrostatic upwind schemes for shallow-water equations. J. Comput. Phys., 231(15):4993-5015, 2012.

[11] C. Berthon, R. Loubère, and V. Michel-Dansac. A Second-Order Well-Balanced Scheme for the Shallow Water Equations with Topography. In Theory, Numerics and Applications of Hyperbolic Problems I, pages 165-177. Springer International Publishing, 2018.

[12] C. Berthon and V. Michel-Dansac. A simple fully well-balanced and entropy preserving scheme for the shallow-water equations. Appl. Math. Lett., 86:284-290, 2018.

[13] C. Berthon, G. Moebs, C. Sarazin-Desbois, and R. Turpault. An asymptotic-preserving scheme for systems of conservation laws with source terms on 2D unstructured meshes. Commun. Appl. Math. Comput. Sci., 11(1):55-77, 2016.

[14] C. Berthon, G. Moebs, and R. Turpault. An asymptotic-preserving scheme for systems of conservation laws with source terms on 2D unstructured meshes. In Finite volumes for complex applications. VII. Methods and theoretical aspects, volume 77 of Springer Proc. Math. Stat., pages 107-115. Springer, Cham, 2014.

[15] F. Bouchut. Nonlinear stability of finite volume methods for hyperbolic conservation laws and well-balanced schemes for sources. Frontiers in Mathematics. Birkhäuser Verlag, Basel, 2004.

[16] J. Burguete, P. Garcí a Navarro, and J. Murillo. Friction term discretization and limitation to preserve stability and conservation in the 1D shallow-water model: application to unsteady irrigation and river flow. Internat. J. Numer. Methods Fluids, 58(4):403-425, 2008.

[17] V. Caleffi and A. Valiani. Well-Balanced Bottom Discontinuities Treatment for High-Order Shallow Water Equations WENO Scheme. J. Eng. Mech., 135(7):684-696, 2009. 
[18] A. Canestrelli, M. Dumbser, A. Siviglia, and E. F. Toro. Well-balanced high-order centered schemes on unstructured meshes for shallow water equations with fixed and mobile bed. Adv. Water Res., 33(3):291$303,2010$.

[19] J. Casper and H. L. Atkins. A finite-volume high-order ENO scheme for two-dimensional hyperbolic systems. J. Comput. Phys., 106(1):62-76, 1993.

[20] M. J. Castro, A. Pardo Milanés, and C. Parés. Well-balanced numerical schemes based on a generalized hydrostatic reconstruction technique. Math. Models Methods Appl. Sci., 17(12):2055-2113, 2007.

[21] M. J. Castro and C. Parés. Well-Balanced High-Order Finite Volume Methods for Systems of Balance Laws. J. Sci. Comput., 82(2), 2020.

[22] M. J. Castro Díaz, J. A. López-García, and C. Parés. High order exactly well-balanced numerical methods for shallow water systems. J. Comput. Phys., 246:242-264, 2013.

[23] C. Chalons, F. Coquel, E. Godlewski, P.-A. Raviart, and N. Seguin. Godunov-type schemes for hyperbolic systems with parameter-dependent source. The case of Euler system with friction. Math. Models Methods Appl. Sci., 20(11):2109-2166, 2010.

[24] P. Chandrashekar and M. Zenk. Well-Balanced Nodal Discontinuous Galerkin Method for Euler Equations with Gravity. J. Sci. Comput, 71(3):1062-1093, 2017.

[25] C. Chen, Z. Lai, R. C. Beardsley, J. Sasaki, J. Lin, H. Lin, R. Ji, and Y. Sun. The March 11, 2011 Tōhoku M9.0 earthquake-induced tsunami and coastal inundation along the Japanese coast: A model assessment. Prog Oceanogr, 123:84-104, 2014.

[26] Y. Cheng, A. Chertock, M. Herty, A. Kurganov, and T. Wu. A New Approach for Designing Moving-Water Equilibria Preserving Schemes for the Shallow Water Equations. J. Sci. Comput., 80(1):538-554, 2019.

[27] V. T. Chow. Open-channel hydraulics. McGraw-Hill civil engineering series. McGraw-Hill, 1959.

[28] S. Clain, S. Diot, and R. Loubère. A high-order finite volume method for systems of conservation lawsMulti-dimensional Optimal Order Detection (MOOD). J. Comput. Phys., 230(10):4028-4050, 2011.

[29] S. Clain and J. Figueiredo. The MOOD method for the non-conservative shallow-water system. Comput. \& Fluids, 145:99-128, 2017.

[30] S. Clain, C. Reis, R. Costa, J. Figueiredo, M. A. Baptista, and J. M. Miranda. Second-order finite volume with hydrostatic reconstruction for tsunami simulation. J. Adv. Model. Earth Syst., 8(4):1691-1713, 2016.

[31] F. Couderc, A. Duran, and J.-P. Vila. An explicit asymptotic preserving low Froude scheme for the multilayer shallow water model with density stratification. J. Comput. Phys., 343:235-270, 2017.

[32] A. Decoene, L. Bonaventura, E. Miglio, and F. Saleri. Asymptotic derivation of the section-averaged shallow water equations for natural river hydraulics. Math. Models Methods Appl. Sci., 19(03):387-417, 2009.

[33] O. Delestre, C. Lucas, P.-A. Ksinant, F. Darboux, C. Laguerre, T.-N.-T. Vo, F. James, and S. Cordier. SWASHES: a compilation of shallow water analytic solutions for hydraulic and environmental studies. Internat. J. Numer. Methods Fluids, 72(3):269-300, 2013. 
[34] V. Desveaux, M. Zenk, C. Berthon, and C. Klingenberg. Well-balanced schemes to capture non-explicit steady states: Ripa model. Math. Comp., 85(300):1571-1602, 2016.

[35] S. Diot, S. Clain, and R. Loubère. Improved detection criteria for the multi-dimensional optimal order detection (MOOD) on unstructured meshes with very high-order polynomials. Comput. \& Fluids, 64:43-63, 2012.

[36] S. Diot, R. Loubère, and S. Clain. The multidimensional optimal order detection method in the threedimensional case: very high-order finite volume method for hyperbolic systems. Internat. J. Numer. Methods Fluids, 73(4):362-392, 2013.

[37] A. Duran, Q. Liang, and F. Marche. On the well-balanced numerical discretization of shallow water equations on unstructured meshes. J. Comput. Phys., 235:565-586, 2013.

[38] J. Figueiredo and S. Clain. Second-order finite volume MOOD method for the shallow water with dry/wet interface. In SYMCOMP 2015, Faro, March 26-27, 2015, Portugal, pages 191-205. ECCOMAS, 2015.

[39] E. Gaburro, M. J. Castro, and M. Dumbser. A well balanced diffuse interface method for complex nonhydrostatic free surface flows. Computers \& Fluids, 175:180-198, 2018.

[40] E. Godlewski and P.-A. Raviart. Hyperbolic systems of conservation laws, volume 3/4 of Mathématiques 63 Applications (Paris) [Mathematics and Applications]. Ellipses, Paris, 1991.

[41] I. Gómez-Bueno, M. J. Castro, and C. Parés. High-order well-balanced methods for systems of balance laws: a control-based approach. Appl. Math. Comput., 394:125820, 2021.

[42] L. Gosse. A well-balanced flux-vector splitting scheme designed for hyperbolic systems of conservation laws with source terms. Comput. Math. Appl., 39(9-10):135-159, 2000.

[43] S. Gottlieb. On high order strong stability preserving Runge-Kutta and multi step time discretizations. $J$. Sci. Comput., 25(1-2):105-128, 2005.

[44] S. Gottlieb and C.-W. Shu. Total variation diminishing Runge-Kutta schemes. Math. Comp., 67(221):73-85, 1998.

[45] S. Gottlieb, C.-W. Shu, and E. Tadmor. Strong stability-preserving high-order time discretization methods. SIAM Rev., 43(1):89-112, 2001.

[46] N. Goutal and F. Maurel. Proceedings of the $2^{\text {nd }}$ Workshop on Dam-Break Wave Simulation. Technical report, Groupe Hydraulique Fluviale, Département Laboratoire National d'Hydraulique, Electricité de France, 1997.

[47] J. M. Greenberg and A.-Y. LeRoux. A well-balanced scheme for the numerical processing of source terms in hyperbolic equations. SIAM J. Numer. Anal., 33(1):1-16, 1996.

[48] A. Harten, P. D. Lax, and B. van Leer. On upstream differencing and Godunov-type schemes for hyperbolic conservation laws. SIAM Rev., 25(1):35-61, 1983.

[49] X. Y. Hu, N. A. Adams, and C.-W. Shu. Positivity-preserving method for high-order conservative schemes solving compressible Euler equations. J. Comput. Phys., 242:169-180, 2013. 
[50] C. Klingenberg, G. Puppo, and M. Semplice. Arbitrary Order Finite Volume Well-Balanced Schemes for the Euler Equations with Gravity. SIAM J. Sci. Comput., 41(2):A695-A721, 2019.

[51] R. J. LeVeque. Numerical methods for conservation laws. Lectures in Mathematics ETH Zürich. Birkhäuser Verlag, Basel, second edition, 1992.

[52] V. Michel-Dansac, C. Berthon, S. Clain, and F. Foucher. A well-balanced scheme for the shallow-water equations with topography. Comput. Math. Appl., 72(3):568-593, 2016.

[53] V. Michel-Dansac, C. Berthon, S. Clain, and F. Foucher. A well-balanced scheme for the shallow-water equations with topography or Manning friction. J. Comput. Phys., 335:115-154, 2017.

[54] V. Michel-Dansac, P. Noble, and J.-P. Vila. Consistent section-averaged shallow water equations with bottom friction. Eur. J. Mech. B. Fluids, 86:123-149, 2021.

[55] I. K. Nikolos and A. I. Delis. An unstructured node-centered finite volume scheme for shallow water flows with wet-dry fronts over complex topography. Comput. Methods Appl. Mech. Engrg., 198(47-48):3723-3750, 2009.

[56] S. Noelle, Y. Xing, and C.-W. Shu. High-order well-balanced finite volume WENO schemes for shallow water equation with moving water. J. Comput. Phys., 226(1):29-58, 2007.

[57] B. Perthame and C.-W. Shu. On positivity preserving finite volume schemes for Euler equations. Numer. Math., 73(1):119-130, 1996.

[58] C. Reis, J. Figueiredo, S. Clain, R. Omira, M. A. Baptista, and J. M. Miranda. Comparison between MUSCL and MOOD techniques in a finite volume well-balanced code to solve SWE. The Tohoku-Oki, 2011 example. Geophys. J. Int., 216(2):958-983, 2018.

[59] S. J. Ruuth. Global optimization of explicit strong-stability-preserving Runge-Kutta methods. Math. Comp., 75(253):183-207, 2006.

[60] G. W. Stewart. Matrix algorithms. Vol. I. Society for Industrial and Applied Mathematics, Philadelphia, PA, 1998. Basic decompositions.

[61] A. Thomann, G. Puppo, and C. Klingenberg. An all speed second order well-balanced IMEX relaxation scheme for the Euler equations with gravity. J. Comput. Phys., 420:109723, 2020.

[62] A. Thomann, M. Zenk, and C. Klingenberg. A second-order positivity-preserving well-balanced finite volume scheme for Euler equations with gravity for arbitrary hydrostatic equilibria. Internat. J. Numer. Methods Fluids, 89(11):465-482, 2019.

[63] E. F. Toro. Riemann solvers and numerical methods for fluid dynamics. A practical introduction. SpringerVerlag, Berlin, third edition, 2009.

[64] B. van Leer. Flux-vector splitting for the Euler equations. In Eighth international conference on numerical methods in fluid dynamics, pages 507-512. Springer, 1982.

[65] Y. Xing. Exactly well-balanced discontinuous Galerkin methods for the shallow water equations with moving water equilibrium. J. Comput. Phys., 257(part A):536-553, 2014. 
[66] A. R. Zarrati, Y. C. Jin, and S. Karimpour. Semianalytical Model for Shear Stress Distribution in Simple and Compound Open Channels. J. Hydraul. Eng., 134(2):205-215, 2008.

[67] F. Zhou, G. Chen, S. Noelle, and H. Guo. A well-balanced stable generalized Riemann problem scheme for shallow water equations using adaptive moving unstructured triangular meshes. Internat. J. Numer. Methods Fluids, 73(3):266-283, 2013. 